\title{
Perverse filtrations, Hilbert schemes, and the $P=W$ conjecture for parabolic Higgs bundles
}

\author{
Junliang Shen and Zili Zhang
}

\begin{abstract}
We prove de Cataldo-Hausel-Migliorini's $P=W$ conjecture in arbitrary rank for parabolic Higgs bundles labeled by the affine Dynkin diagrams $\tilde{A}_{0}, \tilde{D}_{4}, \tilde{E}_{6}, \tilde{E}_{7}$, and $\tilde{E}_{8}$. Our proof relies on the study of the tautological classes on the Hilbert scheme of points on an elliptic surface with respect to the perverse filtration.
\end{abstract}

\section{Introduction}

\subsection{Perverse filtrations}

Let $D_{c}^{b}(Y)$ denote the bounded derived category of constructible sheaves on a nonsingular algebraic variety $Y$. The truncation functor

$$
{ }^{\mathfrak{p}} \tau_{\leqslant k}: D_{c}^{b}(Y) \rightarrow D_{c}^{b}(Y)
$$

with respect to the perverse $t$-structure on $D_{c}^{b}(Y)$ (see [BBD82]) induces a natural morphism

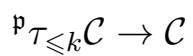

for any object $\mathcal{C} \in D_{c}^{b}(Y)$ and $k \in \mathbb{Z}$.

Let $f: X \rightarrow Y$ be a proper morphism between two irreducible nonsingular algebraic varieties of relative dimension $r$. The morphism

$$
{ }^{\mathfrak{p}} \tau_{\leqslant k} R f_{*} \mathbb{Q}_{X} \rightarrow R f_{*} \mathbb{Q}_{X}
$$

given by (1.1) induces a morphism of (hyper-)cohomology groups,

$$
H^{d-(\operatorname{dim} X-r)}\left(Y,{ }^{\mathfrak{p}} \tau_{\leqslant k}\left(R f_{*} \mathbb{Q}_{X}[\operatorname{dim} X-r]\right)\right) \rightarrow H^{d}(X, \mathbb{Q}) .
$$

Following [deCHM12], we define $P_{k}^{f} H^{d}(X, \mathbb{Q}) \subset H^{d}(X, \mathbb{Q})$ to be the image of $(1.2)$ (the shift $[\operatorname{dim} X-r]$ is to ensure that the perverse filtration is concentrated in the degrees $[0,2 r]$; see Section 2), and we call the increasing filtration

$$
P_{0}^{f} H^{d}(X, \mathbb{Q}) \subset P_{1}^{f} H^{d}(X, \mathbb{Q}) \subset \cdots \subset H^{d}(X, \mathbb{Q})
$$

Received 27 April 2019, accepted in final form 10 January 2021.

2020 Mathematics Subject Classification 14D20, $14 \mathrm{~F} 45$.

Keywords: perverse filtrations, Hilbert schemes, parabolic Higgs bundles.

This journal is (C) Foundation Compositio Mathematica 2021. This article is distributed with Open Access under the terms of the Creative Commons Attribution Non-Commercial License, which permits non-commercial reuse, distribution, and reproduction in any medium, provided that the original work is properly cited. For commercial re-use, please contact the Foundation Compositio Mathematica. 


\section{J. SHen AND Z. ZHANG}

the perverse filtration associated with the map $f$. The perverse filtration (1.3) is called multiplicative if

$$
P_{k}^{f} H^{*}(X, \mathbb{Q}) \cup P_{k^{\prime}}^{f} H^{*}(X, \mathbb{Q}) \subset P_{k+k^{\prime}}^{f} H^{*}(X, \mathbb{Q})
$$

for the cup product $\cup$ and any $k, k^{\prime} \geqslant 0$.

We refer to [BBD82, deCM05, deCM09] for more details about derived categories of constructible sheaves and perverse $t$-structures. See also Section 2 for discussions on perverse filtrations.

\subsection{Hilbert schemes of points on elliptic surfaces}

We first locate tautological cohomology classes for the Hilbert scheme of points on an elliptic surface with respect to the natural perverse filtration. ${ }^{1}$

Let $S$ be an irreducible nonsingular projective surface fibered over a nonsingular curve $C$, that is, $\pi: S \rightarrow C$, such that a general fiber of $\pi$ is an elliptic curve. We assume that $\pi^{[n]}: S^{[n]} \rightarrow C^{(n)}$ is the induced morphism between the Hilbert scheme $S^{[n]}$ of $n$ points on $S$ and the symmetric power $C^{(n)}$. Let $\bar{\pi}^{[n]}$ be the Cartesian product

$$
\bar{\pi}^{[n]}=\pi^{[n]} \times \pi: S^{[n]} \times S \rightarrow C^{(n)} \times C .
$$

THEOREM 1.1. Assume that the perverse filtration associated with $\pi^{[n]}$ is multiplicative for any $n \geqslant 0$. We have

$$
c_{k}\left(\mathcal{O}_{Z_{n}}\right) \in P_{k}^{\bar{\pi}^{[n]}} H^{2 k}\left(S^{[n]} \times S, \mathbb{Q}\right),
$$

where $Z_{n} \subset S^{[n]} \times S$ is the universal subscheme.

It was shown in [LQW02] that the cohomology ring $H^{*}\left(S^{[n]}, \mathbb{Q}\right)$ is generated by the tautological classes - the Künneth factors of $c_{k}\left(\mathcal{O}_{Z_{n}}\right)$ in $H^{*}\left(S^{[n]}, \mathbb{Q}\right)$. Hence Theorem 1.1 describes the perverse filtration associated with $\pi^{[n]}$ via the tautological classes. This plays an essential role in the application of the $P=W$ conjecture; see Sections 1.3 and 1.4 for some special parabolic cases and [deCMS19] for the case of genus 2 and arbitrary rank.

As a corollary of Theorem 1.1, we obtain the following unconditional result for elliptic K3 surfaces.

Corollary 1.2. Let $\pi: S \rightarrow \mathbb{P}^{1}$ be an elliptic $K 3$ surface; then (1.4) holds.

\subsection{Moduli of parabolic Higgs bundles}

Our main motivation for the study of perverse filtrations for Hilbert schemes is the $P=W$ conjecture [deCHM12] for (parabolic) Higgs bundles; see Section 1.4.

In [Gro14], Gröchenig described five infinite families of moduli spaces of parabolic Higgs bundles labeled by the affine Dynkin diagrams $\tilde{A}_{0}, \tilde{D}_{4}, \tilde{E}_{6}, \tilde{E}_{7}$, and $\tilde{E}_{8}$, which extends a result of Gorsky-Nekrasov-Rubtsov [GNR01]. For each Dynkin diagram above, there is attached a sequence of moduli spaces

$$
\left\{M_{n}\right\}_{n \geqslant 1}, \quad \operatorname{dim} M_{n}=2 n,
$$

which are associated with an elliptic curve $E$ with an action of a finite group $\Gamma$. The variety $M_{n}$ can be realized as either the moduli space of rank $n|\Gamma|$ stable Higgs bundles (where $|\Gamma|$ denotes

\footnotetext{
${ }^{1}$ The assumption that the surface admits an elliptic fibration is essential. See Remark 3.3.
} 
the size of the finite group $\Gamma$ ) on the orbifold curve

$$
\mathbb{P}_{\Gamma}=[E / \Gamma],
$$

or the moduli space of certain stable parabolic Higgs bundles on the coarse moduli space of (1.5):

$$
\left[\mathbb{P}_{\Gamma}\right]_{\text {coarse }}= \begin{cases}E, & \tilde{A}_{0} \text { case } \\ \mathbb{P}^{1}, & \text { the others }\end{cases}
$$

see [Gro14] for more details. Gröchenig further showed that $M_{1}$ is a nonsingular surface elliptically fibered over the affine line, $\pi_{1}: M_{1} \rightarrow \mathbb{C}$, and $M_{n}$ is the Hilbert scheme of $n$-points on $M_{1}$ with the Hitchin fibration

$$
\pi_{n}: M_{n}=M_{1}^{[n] \stackrel{\pi_{1}^{[n]}}{\longrightarrow}} \mathbb{C}^{n}
$$

In Section 4, we introduce a canonical decomposition

$$
H^{*}\left(M_{n}, \mathbb{Q}\right)=\bigoplus_{i \geqslant 0} G_{i} H^{*}\left(M_{n}, \mathbb{Q}\right)
$$

splitting the perverse filtration on $H^{*}\left(M_{n}, \mathbb{Q}\right)$ associated with $\pi_{n}$, that is,

$$
P_{k}^{\pi_{n}} H^{*}\left(M_{n}, \mathbb{Q}\right)=\bigoplus_{i \leqslant k} G_{i} H^{*}\left(M_{n}, \mathbb{Q}\right) .
$$

We call such a splitting the perverse decomposition.

For an orbifold (or a Deligne-Mumford stack) $\mathcal{X}$, we define $\mathcal{I} \mathcal{X}$ to be the corresponding inertia stack equipped with the canonical morphism $\mathcal{I} \mathcal{X} \rightarrow \mathcal{X}$. We refer to [Ols16, Chapter 8] for the definition and basic properties of inertia stacks. We use $H_{\text {orb }}^{*}\left(\mathbb{P}_{\Gamma}, \mathbb{Q}\right)$ to denote the cohomology $H^{*}\left(\mathcal{I} \mathbb{P}_{\Gamma}, \mathbb{Q}\right)$.

A universal family $(\mathcal{E}, \sigma)$ on $M_{n} \times \mathbb{P}_{\Gamma}$ is called normalized if

$$
\left.c_{1}(\mathcal{E})\right|_{M_{n} \times p}=0
$$

with $p \in \mathbb{P}_{\Gamma}$ a point. The existence of such a universal family for $M_{n}$ is guaranteed by the proof of [Gro14, Theorem 5.1], where it is given by the universal family of the Hilbert scheme $M_{1}^{[n]}$ and a Fourier-Mukai transform. (In general, a universal family on $M_{n} \times \mathbb{P}_{\Gamma}$ is not unique. The difference of two universal families is given by the pullback of a line bundle from $M_{n}$. However, in our case, we actually work with a canonical universal family induced by the geometry of Hilbert schemes and the Poincaré line bundle for elliptic curves; see Section 4 for more details.) For notational convenience, all universal families are assumed to be normalized throughout the paper. In particular, their Chern characters are canonically defined.

The following theorem concerns the precise locations of the tautological classes on $M_{n}$ with respect to the perverse decomposition (1.6). The tautological classes (1.7) below are defined following the convention of Section 1.7 by integrating classes $\alpha \in H^{*}\left(\mathcal{I P}_{\Gamma}, \mathbb{Q}\right)$.

Theorem 1.3. Let $(\mathcal{E}, \sigma)$ be the universal Higgs bundle over $M_{n} \times \mathcal{I P}_{\Gamma}$ pulled back from the universal family on $M_{n} \times \mathbb{P}_{\Gamma}$. Then the following statements hold:

(i) The tautological classes

$$
\int_{\alpha} c_{k}(\mathcal{E}) \in H^{*}\left(M_{n}, \mathbb{Q}\right), \quad \alpha \in H_{\text {orb }}^{*}\left(\mathbb{P}_{\Gamma}, \mathbb{Q}\right)
$$

generate the cohomology ring $H^{*}\left(M_{n}, \mathbb{Q}\right)$. 


\section{J. SHen AND Z. ZHANG}

(ii) We have

$$
\int_{\alpha} c_{k}(\mathcal{E}) \in G_{k} H^{*}\left(M_{n}, \mathbb{Q}\right) \subset P_{k}^{\pi_{n}} H^{*}\left(M_{n}, \mathbb{Q}\right)
$$

for any $\alpha \in H_{\text {orb }}^{*}\left(\mathbb{P}_{\Gamma}, \mathbb{Q}\right)$.

\subsection{The $P=W$ conjecture}

Simpson established in [Sim92] the nonabelian Hodge theorem for a curve of genus $\geqslant 2$ and the reductive group $\mathrm{GL}_{n}$, which gives a canonical diffeomorphism between the moduli space $\mathcal{M}_{\text {Dol }}$ of rank $n$ stable Higgs bundles and the corresponding character variety $\mathcal{M}_{B}$ of rank $n$ irreducible local systems.

A striking phenomenon was discovered by de Cataldo, Hausel, and Migliorini in [deCHM12], namely that the canonical isomorphism

$$
H^{*}\left(\mathcal{M}_{B}, \mathbb{Q}\right)=H^{*}\left(\mathcal{M}_{\text {Dol }}, \mathbb{Q}\right)
$$

induced by Simpson's correspondence is expected to identify the weight filtration $W_{2} \bullet H^{*}\left(\mathcal{M}_{B}, \mathbb{Q}\right)$ and the perverse filtration $P_{\bullet} H^{*}\left(\mathcal{M}_{\text {Dol }}, \mathbb{Q}\right)$ associated with the Hitchin fibration, that is,

$$
W_{2 k} H^{*}\left(\mathcal{M}_{B}, \mathbb{Q}\right)=W_{2 k+1} H^{*}\left(\mathcal{M}_{B}, \mathbb{Q}\right)=P_{k} H^{*}\left(\mathcal{M}_{\text {Dol }}, \mathbb{Q}\right), \quad k \geqslant 0 .
$$

Such a phenomenon is referred to as "the $P=W$ conjecture." The original $P=W$ conjecture was verified for $n=2$ in [deCHM12], while the $n \geqslant 3$ cases are still open.

The main purpose of this paper is to provide a proof of the $P=W$ conjecture for the moduli spaces $M_{n}$ of parabolic Higgs bundles associated with the affine Dynkin diagrams $\tilde{A}_{0}, \tilde{D}_{4}, \tilde{E}_{6}, \tilde{E}_{7}$, and $\tilde{E}_{8}$, as described in Section 1.3.

Let $\mathcal{M}_{n}^{\prime}$ be the character variety of stable parabolic local systems associated with $M_{n}$ via the correspondence [Sim90]. The parabolic nonabelian Hodge theorem was proven in [Biq97, BGM20]; it induces a canonical isomorphism of the cohomology groups

$$
H^{*}\left(\mathcal{M}_{n}^{\prime}, \mathbb{Q}\right)=H^{*}\left(M_{n}, \mathbb{Q}\right) \text {. }
$$

Theorem 1.4. The $P=W$ conjecture holds for the five families of moduli spaces of parabolic Higgs bundles/local systems labeled by the Dynkin diagrams $\tilde{A}_{0}, \tilde{D}_{4}, \tilde{E}_{6}, \tilde{E}_{7}$, and $\tilde{E}_{8}$, i.e., under the identification (1.8) we have

$$
W_{2 k} H^{*}\left(\mathcal{M}_{n}^{\prime}, \mathbb{Q}\right)=W_{2 k+1} H^{*}\left(\mathcal{M}_{n}^{\prime}, \mathbb{Q}\right)=P_{k} H^{*}\left(M_{n}, \mathbb{Q}\right), \quad k \geqslant 0 .
$$

In fact, we prove a refinement of Theorem 1.4 in Section 4 . For every character variety $\mathcal{M}_{n}^{\prime}$ above, we consider the following sub-vector space of $H^{d}\left(\mathcal{M}_{n}^{\prime}, \mathbb{Q}\right)$ :

$$
{ }^{k} \operatorname{Hdg}^{d}\left(\mathcal{M}_{n}^{\prime}\right)=W_{2 k} H^{d}\left(\mathcal{M}_{n}^{\prime}, \mathbb{Q}\right) \cap F^{k} H^{d}\left(\mathcal{M}_{n}^{\prime}, \mathbb{C}\right) .
$$

Using an argument similar to that in [She17], we show in Section 4.5 that the cohomology of the character variety $\mathcal{M}_{n}^{\prime}$ admits a canonical decomposition

$$
H^{*}\left(\mathcal{M}_{n}^{\prime}, \mathbb{Q}\right)=\bigoplus_{k, d}^{k} \operatorname{Hdg}^{d}\left(\mathcal{M}_{n}^{\prime}\right)
$$

The decomposition (1.9) is then shown to match the perverse decomposition (1.6), which implies Theorem 1.4 as a conclusion.

Our results provide examples of moduli of parabolic Higgs bundles where the $P=W$ conjecture holds for arbitrary rank. The construction of the perverse decomposition (1.6) and Theorem 1.3 play an essential role in the proof. 


\section{Perverse filtrations, Hilbert schemes, and $P=W$}

\subsection{Relation to other work}

An interesting phenomenon has been found in [deCHM13] and extended in [Zha17, Section 4.4], namely that the perverse filtration for $M_{n}=M_{1}^{[n]}$ matches the weight filtration for the Hilbert scheme $\mathcal{M}_{1}^{\prime}{ }^{[n]}$. However, this exchange of filtrations is not " $P=W$ " since a character variety is an affine variety which cannot be realized as the Hilbert scheme of points.

As generalizations of the Higgs moduli spaces studied in [deCHM13], the moduli spaces considered in this paper are quite different from the ones treated in [deCHM12]. In the latter case, due to Ngô's support theorem, most of the perverse sheaves showing up in the decomposition theorem are supported on all of the target space of the Hitchin map. However, in the cases of Theorem 1.4, every stratum in $\mathbb{C}^{n}=\mathbb{C}^{(n)}$ of the Hitchin map $\pi_{n}: M_{n} \rightarrow \mathbb{C}^{(n)}$ contributes as a support in the decomposition theorem.

As an application of our result, we provide closed formulas for the mixed Hodge numbers of certain character varieties. This is discussed in Section 4.7. In particular, our formulas match the conjecture of Hausel, Letellier, and Rodriguez-Villegas [HLR11, Conjecture 1.2.1] numerically.

In the recent work [deCMS19], the tautological classes for the Hilbert scheme of points on an abelian surface play a crucial role in the proof of the $P=W$ conjecture for $\mathrm{GL}_{n}$ and a genus 2 curve, although at a first glance, the geometry of these Higgs moduli spaces is very different from that of the Hilbert schemes of points.

\subsection{Idea of the proof}

Our proof of the $P=W$ identities in this paper is based on the understanding of the cohomology rings of both moduli spaces $M_{n}$ and $\mathcal{M}_{n}^{\prime}$. Both cohomology rings are generated by the tautological classes in the sense of Theorem 1.3. Hence the filtrations $P_{\bullet} H^{*}\left(M_{n}, \mathbb{Q}\right)$ and $W_{2} \bullet H^{*}\left(\mathcal{M}_{n}^{\prime}, \mathbb{Q}\right)$ can be naturally identified by showing that both filtrations admit natural multiplicative splittings, and the tautological classes lie in the same piece of the decompositions. We refer to Section 4.1 for a detailed summary of these steps.

We note that two different structures of the cohomology of $M_{n}$ are essentially used. Göttsche and Soergel [GS93] introduce a basis of $H^{*}\left(M_{n}, \mathbb{Q}\right)$, which has combinatorial nature and relies heavily on the description of $M_{n}$ as the Hilbert scheme $M_{1}^{[n]}$. This basis is applied to calculate the perverse filtration explicitly for $M_{n}$ and prove the multiplicativity for the perverse filtration. However, since the character variety $\mathcal{M}_{n}^{\prime}$ is not a Hilbert scheme of points, we lose track of Göttsche-Soergel's combinatorial classes on $\mathcal{M}_{n}^{\prime}$ under the canonical identification

$$
H^{*}\left(M_{n}, \mathbb{Q}\right)=H^{*}\left(\mathcal{M}_{n}^{\prime}, \mathbb{Q}\right) .
$$

Alternatively, we achieve the comparison by considering the tautological classes induced by the universal families. We study the tautological classes via special geometries of Hilbert schemes, which allows us to calculate these classes with respect to the perverse filtration without analyzing the topology of the Hitchin fibration $\pi_{n}: M_{n} \rightarrow \mathbb{C}^{n}$.

\subsection{Conventions}

Throughout the paper, we work over the complex numbers $\mathbb{C}$. For a nonsingular projective variety (or Deligne-Mumford stack) $X$, a quasi-projective variety $Y$, and an object $\mathcal{F} \in D^{b}(X \times Y)$, we say that a correspondence $g: H^{*}(X, \mathbb{Q}) \rightarrow H^{*}(Y, \mathbb{Q})$ is induced by $\mathcal{F}$ if $g$ is given by

$$
g(\alpha)=\operatorname{pr}_{Y *}\left(\operatorname{pr}_{X}^{*}\left(\alpha \cup \operatorname{td}{ }_{X}\right) \cup \operatorname{ch}(\mathcal{F})\right) .
$$




\section{J. SHen AND Z. ZHANG}

For $\gamma \in H^{*}(X, \mathbb{Q})$ and $\alpha \in H^{*}(X \times Y, \mathbb{Q})$, we use $\int_{\gamma} \alpha$ to denote the class

$$
\operatorname{pr}_{Y *}\left(\operatorname{pr}_{X}^{*} \gamma \cup \alpha\right) \in H^{*}(Y, \mathbb{Q}) \text {. }
$$

\section{Perverse decompositions for Hilbert schemes}

\subsection{Perverse filtrations}

We define the perverse filtration associated with any proper morphism $f: X \rightarrow Y$ between nonsingular varieties.

Let

$$
r(f)=\operatorname{dim} X \times_{Y} X-\operatorname{dim} X
$$

be the defect of semismallness. In particular, we have $r(f)=r$ when $f$ has relative dimension $r$, and we have $r(f)=0$ if $f$ is semismall.

As in Section 1.1, we define the perverse filtration $P_{k}^{f} H^{*}(X, \mathbb{Q})$ by

$$
P_{k}^{f} H^{d}(X, \mathbb{Q}):=\operatorname{Im}\left\{H^{d-\operatorname{dim} X+r(f)}\left(X,{ }^{\mathfrak{p}} \tau_{\leqslant k}\left(R f_{*} \mathbb{Q}_{X}[\operatorname{dim} X-r(f)]\right)\right) \rightarrow H^{d}(X, \mathbb{Q})\right\} .
$$

Once we choose a decomposition

$$
R f_{*} \mathbb{Q}_{X}[\operatorname{dim} X-r(f)] \cong \bigoplus_{i=0}^{2 r(f)} \mathcal{P}_{i}[-i] \in D_{c}^{b}(Y)
$$

with $\mathcal{P}_{i}$ perverse sheaves on $Y$ (see [BBD82, deCM09]), the perverse filtration associated with the morphism $f$ can be computed as

$$
P_{k}^{f} H^{*}(X, \mathbb{Q})=\operatorname{Im}\left\{H^{*-\operatorname{dim} X+r(f)}\left(\oplus_{i=0}^{k} \mathcal{P}_{i}[-i]\right) \rightarrow H^{*}(X, \mathbb{Q})\right\} .
$$

In general, a perverse filtration does not allow a natural splitting since the decomposition (2.1) is dependent on the choice of an isomorphism which is not canonical.

We define the perversity $\mathfrak{p}^{f}(\alpha)$ of a class $\alpha \in H^{d}(X, \mathbb{Q})$ to be the integer $k$ satisfying $\alpha \in$ $P_{k}^{f} H^{d}(X, \mathbb{Q})$ and $\alpha \notin P_{k-1}^{f} H^{d}(X, \mathbb{Q})$. Since the perverse filtration is concentrated in the degrees $[0,2 r(f)],{ }^{2}$ we have

$$
0 \leqslant \mathfrak{p}^{f}(\alpha) \leqslant 2 r(f)
$$

for any class $\alpha \in H^{*}(X, \mathbb{Q})$.

Let $f_{1}: X_{1} \rightarrow Y_{1}$ and $f_{2}: X_{2} \rightarrow Y_{2}$ be proper morphisms between nonsingular quasi-projective varieties. We recall the following proposition from [Zha17] concerning the perverse filtration associated with the product

$$
f_{1} \times f_{2}: X_{1} \times X_{2} \rightarrow Y_{1} \times Y_{2} .
$$

Proposition 2.1 ([Zha17, Proposition 2.1]). For $\alpha_{1} \in H^{*}\left(X_{1}, \mathbb{Q}\right)$ and $\alpha_{2} \in H^{*}\left(X_{2}, \mathbb{Q}\right)$, we have

$$
\mathfrak{p}^{f_{1} \times f_{2}}\left(\alpha_{1} \otimes \alpha_{2}\right)=\mathfrak{p}^{f_{1}}\left(\alpha_{1}\right)+\mathfrak{p}^{f_{2}}\left(\alpha_{2}\right) \text {. }
$$

\subsection{Perverse decompositions}

In this section, we introduce perverse decompositions for symmetric powers and Hilbert schemes of points associated with a surface, which split the perverse filtrations on the corresponding cohomology groups. ${ }^{2}$ We recall that a filtration on a vector space is concentrated in the degrees $[a, b]$ if the $k$ th graded piece of the
filtration is empty when $k \notin[a, b]$. 


\section{Perverse filtrations, Hilbert schemes, and $P=W$}

Let $\pi: S \rightarrow C$ be a proper surjective morphism from a nonsingular quasi-projective surface to a nonsingular curve. Then $r(\pi)=1$, and $H^{*}(S, \mathbb{Q})$ admits a perverse filtration of length 2,

$$
P_{0}^{\pi} H^{*}(S, \mathbb{Q}) \subset P_{1}^{\pi} H^{*}(S, \mathbb{Q}) \subset P_{2}^{\pi} H^{*}(S, \mathbb{Q})=H^{*}(S, \mathbb{Q})
$$

Throughout Section 3, we fix a decomposition

$$
H^{*}(S, \mathbb{Q})=\bigoplus_{i \geqslant 0} G_{i} H^{*}(S, \mathbb{Q})
$$

splitting the perverse filtration (2.3); that is, the decomposition (2.2) satisfies

$$
P_{k}^{\pi} H^{*}(S, \mathbb{Q})=\bigoplus_{i \leqslant k} G_{i} H^{*}(S, \mathbb{Q})
$$

for $k \in\{0,1,2\}$. In particular, every class in $G_{i} H^{*}(S, \mathbb{Q})$ has perversity $i$.

We define the decomposition of $H^{*}\left(S^{n}, \mathbb{Q}\right)$ to be

$$
G_{k} H^{*}\left(S^{n}, \mathbb{Q}\right)=\left\langle\alpha_{1} \otimes \cdots \otimes \alpha_{n} ; \alpha_{i} \in G_{k_{i}} H^{*}(S, \mathbb{Q}), \sum_{i} k_{i}=k\right\rangle .
$$

Due to Proposition 2.1, this decomposition splits the perverse filtration of $\pi^{n}: S^{n} \rightarrow C^{n}$. Taking the $\mathfrak{S}_{n}$-invariant part yields the perverse decomposition for the symmetric power $S^{(n)}$ associated with $\pi^{(n)}: S^{(n)} \rightarrow C^{(n)}$,

$$
H^{*}\left(S^{(n)}, \mathbb{Q}\right)=\bigoplus_{i \geqslant 0} G_{i} H^{*}\left(S^{(n)}, \mathbb{Q}\right) .
$$

The perverse decomposition for the product $S^{\left(n_{1}\right)} \times S^{\left(n_{2}\right)} \times \cdots \times S^{\left(n_{k}\right)}$ is then defined in a similar way using the Künneth decomposition.

We consider the Hilbert scheme $S^{[n]}$ of $n$ points on $S$, which is a $2 n$-dimensional nonsingular variety parametrizing length $n 0$-dimensional subschemes in $S$. Rich structures of the cohomology of the Hilbert scheme of points have been found and studied intensively in the last decades; see [Göt90, GS93, Nak97, Leh99, LS03, LQW02]. Now we assume that $\pi^{[n]}: S^{[n]} \rightarrow C^{(n)}$ is the natural morphism associated with $\pi$. For a partition

$$
\nu=1^{a_{1}} 2^{a_{2}} \cdots n^{a_{n}}
$$

of $n$, we use $S^{(\nu)}$ to denote the variety $S^{\left(a_{1}\right)} \times S^{\left(a_{2}\right)} \times \cdots \times S^{\left(a_{n}\right)}$. By [GS93], the cohomology group $H^{d}\left(S^{[n]}, \mathbb{Q}\right)$ admits a canonical decomposition

$$
H^{d}\left(S^{[n]}, \mathbb{Q}\right)=\bigoplus_{\nu} H^{d+2 l(\nu)-2 n}\left(S^{(\nu)}, \mathbb{Q}\right)
$$

where $\nu$ runs through all partitions of $n$ and $l(\nu)$ is the length of $\nu$. We define

$$
G_{k} H^{d}\left(S^{[n]}, \mathbb{Q}\right)=\bigoplus_{\nu} G_{k+l(\nu)-n} H^{d+2 l(\nu)-2 n}\left(S^{(\nu)}, \mathbb{Q}\right)
$$

to be the sub-vector space of $H^{d}\left(S^{[n]}, \mathbb{Q}\right)$ under the identification $(2.4)$. The following proposition is obtained from [Zha17, Proposition 4.12].

Proposition 2.2. The decomposition

$$
H^{*}\left(S^{[n]}, \mathbb{Q}\right)=\bigoplus_{i \geqslant 0} G_{i} H^{*}\left(S^{[n]}, \mathbb{Q}\right)
$$

defined above splits the perverse filtration associated with $\pi^{[n]}: S^{[n]} \rightarrow C^{(n)}$. 


\section{J. SHEN AND Z. ZHANG}

We have constructed perverse decompositions on the cohomology groups $H^{*}\left(S^{[n]}, \mathbb{Q}\right)$. Again, by Proposition 2.1, we obtain perverse decompositions for the product of Hilbert schemes

$$
S^{\left[n_{1}\right]} \times S^{\left[n_{2}\right]} \times \cdots \times S^{\left[n_{k}\right]} .
$$

\subsection{Nested Hilbert schemes}

We consider the nested Hilbert scheme

$$
S^{[n, n+1]}=\left\{(\xi, \eta): \xi \in S^{[n+1]}, \eta \in S^{[n]}, \xi \subset \eta\right\} \subset S^{[n+1]} \times S^{[n]},
$$

which is a nonsingular and irreducible variety of dimension $2 n+2$; see [Che98]. In this section, we extend perverse decompositions to the nested Hilbert schemes associated with $\pi: S \rightarrow C$; this extension plays a crucial role in the proofs of Theorems 1.1 and 1.4.

The variety $S^{[n, n+1]}$ admits the following natural morphisms

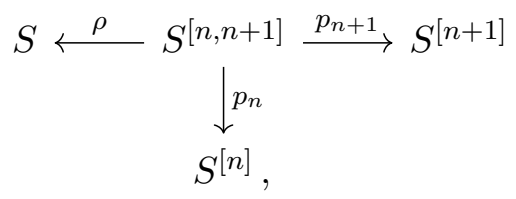

where $p_{n}$ and $p_{n+1}$ are the two projections and $\rho$ sends $(\xi, \eta)$ to the point $\{\eta \backslash \xi\} \in S$. The morphism

$$
q_{n}=\left(p_{n}, \rho\right): S^{[n, n+1]} \rightarrow S^{[n]} \times S
$$

is realized as the blow-up of the codimension 2 incidence $Z_{n} \subset S^{[n]} \times S$ with the exceptional divisor

$$
E_{n+1}=\left\{(\xi, \eta) \in S^{[n+1, n]}: \operatorname{Supp}(\xi)=\operatorname{Supp}(\eta)\right\} \subset S^{[n, n+1]} .
$$

Let $g_{n}: S^{[n, n+1]} \rightarrow S^{(n)} \times S$ be the composition

$$
S^{[n, n+1]} \stackrel{q_{n}}{\longrightarrow} S^{[n]} \times S \rightarrow S^{(n)} \times S,
$$

and let

$$
\bar{g}_{n}=\left(\pi^{(n)} \times \pi\right) \circ g_{n}: S^{[n, n+1]} \rightarrow C^{(n)} \times C .
$$

We define

$$
S^{(\nu, j)}= \begin{cases}S^{(\nu)} \times S, & j=0, \\ S^{\left(\nu^{b}\right)} \times S, & j>0 \text { and } a_{j}>0, \\ \varnothing, & j>0 \text { and } a_{j}=0,\end{cases}
$$

where $\nu=1^{a_{1}} \cdots n^{a_{n}}$ is a partition of $n$ and $\nu^{b}$ is defined as a partition of $n-j$ obtained from $\nu$ by reducing $a_{j}$ by 1 . This definition matches the one in [deCM04, Section 3.3]. In particular, $S^{(\nu, j)}$ is a Cartesian product of symmetric powers of $S$. Hence the cohomology of $S^{(\nu, j)}$ carries a natural perverse decomposition as defined in Section 2.2,

$$
H^{*}\left(S^{(\nu, j)}, \mathbb{Q}\right)=\bigoplus_{k \geqslant 0} G_{k} H^{*}\left(S^{(\nu, j)}, \mathbb{Q}\right)
$$

By [deCM04, Theorem 3.3.1], there is a canonical isomorphism

$$
H^{d}\left(S^{[n, n+1]}, \mathbb{Q}\right)=\bigoplus_{\nu, j} H^{d-2 m(\nu, j)}\left(S^{(\nu, j)}, \mathbb{Q}\right),
$$


where

$$
m(\nu, j)= \begin{cases}n-l(\nu), & j=0 \\ n+1-l(\nu), & j>0\end{cases}
$$

We define the following decomposition of $H^{d}\left(S^{[n, n+1]}, \mathbb{Q}\right)$ under the identification $(2.7)$ :

$$
G_{k} H^{d}\left(S^{[n, n+1]}, \mathbb{Q}\right)=\bigoplus_{\nu, j} G_{k-m(\nu, j)} H^{d-2 m(\nu, j)}\left(S^{(\nu, j)}, \mathbb{Q}\right) .
$$

Since $S^{(\nu, j)}$ is a product of symmetric powers of $S$ for every $(\nu, j)$, the method in [Zha17, Proposition 4.12] together with the decomposition theorem [deCM04, Theorem 3.3.1] implies the following proposition.

Proposition 2.3. The decomposition

$$
H^{*}\left(S^{[n, n+1]}, \mathbb{Q}\right)=\bigoplus_{i \geqslant 0} G_{i} H^{*}\left(S^{[n, n+1]}, \mathbb{Q}\right)
$$

defined above splits the perverse filtration associated with $\bar{g}_{n}: S^{[n, n+1]} \rightarrow C^{(n)} \times C$.

\subsection{Functoriality}

The natural morphisms

$$
q_{n}: S^{[n, n+1]} \rightarrow S^{[n]} \times S \quad \text { and } \quad p_{n+1}: S^{[n, n+1]} \rightarrow S^{[n+1]}
$$

are generically finite. In the following, we prove some functoriality results concerning the morphisms $q_{n}$ and $p_{n+1}$ with respect to the perverse decompositions constructed in Sections 2.2 and 2.3.

Proposition 2.4. Let $q_{n}^{*}: H^{*}\left(S^{[n]} \times S, \mathbb{Q}\right) \rightarrow H^{*}\left(S^{[n, n+1]}, \mathbb{Q}\right)$ be the pullback morphism. We have

$$
q_{n}^{*}\left(G_{k} H^{*}\left(S^{[n]} \times S, \mathbb{Q}\right)\right) \subset G_{k} H^{*}\left(S^{[n, n+1]}, \mathbb{Q}\right) .
$$

Proof. We define $W$ and $\widetilde{W}$ to make the following diagram as fibered products:

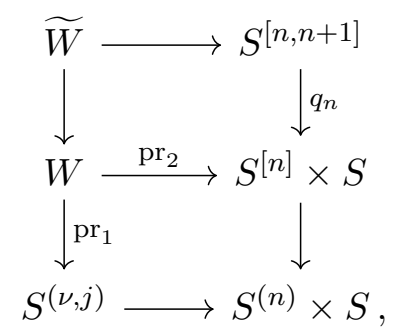

where $S^{(\nu, j)} \rightarrow S^{(n)} \times S$ is the natural morphism to the corresponding stratum [deCM04, Section 3].

On one hand, by [deCM02, Theorem 5.4.1] and the Künneth decomposition, there is a canonical decomposition

$$
H^{*}\left(S^{[n]} \times S, \mathbb{Q}\right)=\bigoplus_{\nu} H^{*}\left(S^{(\nu)} \times S, \mathbb{Q}\right),
$$

where the direct summands are canonically identified with the image of the correspondences

$$
W_{*}=\operatorname{pr}_{2 *} \operatorname{pr}_{1}^{*}: H^{*}\left(S^{(\nu)} \times S, \mathbb{Q}\right)=H^{*}\left(S^{(\nu, 0)}, \mathbb{Q}\right) \rightarrow H^{*}\left(S^{[n]} \times S, \mathbb{Q}\right) .
$$




\section{J. SHEN AND Z. ZHANG}

On the other hand, by [deCM04, Theorem 3.3.1], there is a canonical decomposition

$$
H^{*}\left(S^{[n, n+1]}, \mathbb{Q}\right)=\bigoplus_{\nu, j} H^{*}\left(S^{(\nu, j)}, \mathbb{Q}\right),
$$

where the direct summands are identified with the images of the correspondences

$$
\widetilde{W}_{*}: H^{*}\left(S^{(\nu, j)}, \mathbb{Q}\right) \rightarrow H^{*}\left(S^{[n, n+1]}, \mathbb{Q}\right) .
$$

Since $S^{(\nu)} \times S=S^{(\nu, 0)}$, we obtain a commutative diagram

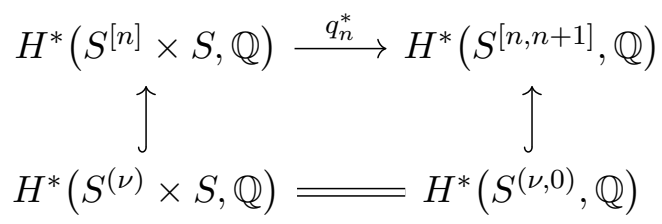

for each partition $\nu$ of $n$. In particular, $q_{n}^{*}$ preserves the perverse decompositions.

Proposition 2.5. Let $p_{n+1 *}: H^{*}\left(S^{[n, n+1]}, \mathbb{Q}\right) \rightarrow H^{*}\left(S^{[n+1]}, \mathbb{Q}\right)$ be the Gysin pushforward. Then we have

$$
p_{n+1 *}\left(G_{k} H^{*}\left(S^{[n, n+1]}, \mathbb{Q}\right)\right) \subset G_{k} H^{*}\left(S^{[n+1]}, \mathbb{Q}\right) .
$$

Proof. Consider the commutative diagram

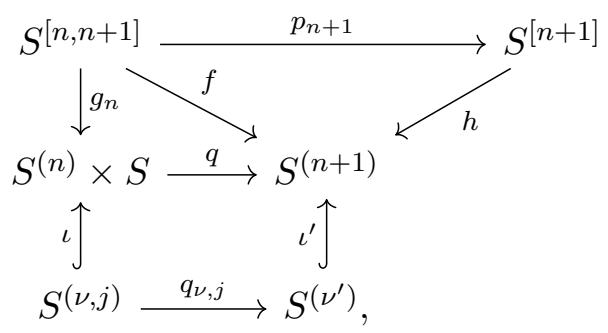

where $\nu=1^{a_{1}} \cdots n^{a_{n}}$ is a partition of $n$ and

$$
\nu^{\prime}= \begin{cases}1^{a_{1}+1} 2^{a_{2}} \cdots n^{a_{n}}, & j=0 \\ 1^{a_{1}} \cdots j^{a_{j}-1}(j+1)^{a_{j+1}+1} \cdots n^{a_{n}}, & j>0 .\end{cases}
$$

By [deCM04, Theorem 3.3.1], we have

$$
R g_{n_{*}} \mathbb{Q}_{S[n, n+1]}=\bigoplus_{\nu, j} \iota_{*} \mathbb{Q}_{S^{(\nu, j)}}[-2 m(\nu, j)]
$$

Since $q \circ \iota: S^{(\nu, j)} \rightarrow S^{(n+1)}$ is a finite map, the object $q_{*} \iota_{*} \mathbb{Q}_{S^{(\nu, j)}}[m(\nu, j)]$ is a perverse sheaf supported on the locus $\iota^{\prime}\left(S^{\left(\nu^{\prime}\right)}\right) \subset S^{(n+1)}$, where $\iota^{\prime}: S^{\left(\nu^{\prime}\right)} \rightarrow S^{(n+1)}$ is the natural map to the corresponding stratum as in [GS93]. We compare the following two decompositions of perverse sheaves on $S^{(n+1)}$,

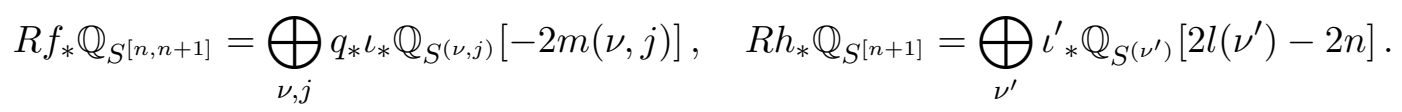

Since simple perverse sheaves with different irreducible supports do not have morphisms between them, we obtain that the pushforward morphism

$$
R f_{*} \mathbb{Q}_{S[n, n+1]} \rightarrow R h_{*} \mathbb{Q}_{S^{[n+1]}}
$$


splits canonically to $q_{*} \iota_{*} \mathbb{Q}_{S(\nu, j)} \rightarrow \iota_{*}^{\prime} \mathbb{Q}_{S^{\left(\nu^{\prime}\right)}}$ for every partition $\nu$ and $j \geqslant 0$. Since the pushforward $p_{n+1 *}: H^{*}\left(S^{[n, n+1]}, \mathbb{Q}\right) \rightarrow H^{*}\left(S^{[n+1]}, \mathbb{Q}\right)$ is induced by the morphism (2.8) of sheaves, we conclude that $p_{n+1 *}$ is given by $\left(q_{\nu, j}\right)_{*}: H^{*}\left(S^{(\nu, j)}, \mathbb{Q}\right) \rightarrow H^{*}\left(S^{\left(\nu^{\prime}\right)}, \mathbb{Q}\right)$ under the decompositions (2.4) and (2.7) for every partition $\nu$ and $j \geqslant 0$.

Finally, since the morphism $q_{\nu, j}$ is finite, the induced pushforward $\left(q_{\nu, j}\right)_{*}$ preserves the perverse decomposition. This completes the proof.

Although the perverse decompositions depend on a choice of (2.3), the perverse filtrations are canonical. The following corollary concerns the functoriality of perverse filtrations.

Corollary 2.6. We have

$$
\begin{aligned}
q_{n}^{*}\left(P_{k}^{\bar{\pi}^{[n]}} H^{*}\left(S^{[n]} \times S, \mathbb{Q}\right)\right) & \subset P_{k}^{\bar{g}_{n}} H^{*}\left(S^{[n, n+1]}, \mathbb{Q}\right), \\
p_{n+1 *}\left(P_{k}^{\bar{g}_{n}} H^{*}\left(S^{[n, n+1]}, \mathbb{Q}\right)\right) & \subset P_{k}^{\pi^{[n+1]}} H^{*}\left(S^{[n+1]}, \mathbb{Q}\right) .
\end{aligned}
$$

In general, for a commutative diagram

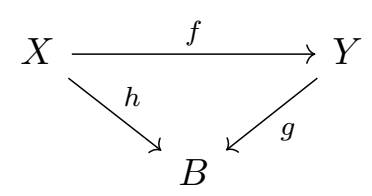

with all varieties nonsingular and all morphisms proper, the perverse filtration $P_{k}^{g} H^{*}(Y, \mathbb{Q})$ (respectively, $P_{k}^{h} H^{*}(X, \mathbb{Q})$ ) may not be preserved by the pullback $f^{*}$ (respectively, the pushforward $f_{*}$ ); see Example 2.7 below. Hence Corollary 2.6 relies on the geometry of (nested) Hilbert schemes.

ExAmple 2.7. (i) In the diagram (2.9), we let $X=\mathrm{Bl}_{\mathrm{pt}} \mathrm{P}^{3}$ and $Y=B=\mathbb{P}^{3}$. Assume that $f, g, h$ are the natural maps. Then we have $\mathfrak{p}^{h}([X])=1$ and $\mathfrak{p}^{g}([Y])=0$; see [Zha17, Example 1.5]. Hence $f^{*} P_{0}^{g} H^{0}(Y, \mathbb{Q}) \not \subset P_{0}^{h} H^{0}(X, \mathbb{Q})$.

(ii) Let $X=B=$ pt and $Y=\mathbb{P}^{1}$. Assume that $f, g, h$ are the natural maps. We have $\mathfrak{p}^{h}([\mathrm{pt}])=0$ and $\mathfrak{p}^{g}([\mathrm{pt}])=2$, and, in particular, $f_{*} P_{0}^{h} H^{*}(X, \mathbb{Q}) \not \subset P_{0}^{g} H^{*}(Y, \mathbb{Q})$.

\subsection{Strong multiplicativity of the perverse decomposition}

In this section, we prove the strong multiplicativity with respect to the canonical perverse decomposition defined in Section 2.2 for the Hilbert scheme of points on a surface with numerically trivial canonical divisor. This is a variant of [Zha17, Theorems 4.18 and 5.6], where only perverse filtrations are considered. Since the proofs are similar, we will give a sketch and focus on pointing out the differences.

Let $\pi: S \rightarrow C$ be a proper surjective morphism from a nonsingular quasi-projective surface to a nonsingular quasi-projective curve, and let

$$
H^{*}(S, \mathbb{Q})=\bigoplus_{i} G_{i} H^{*}(S, \mathbb{Q})
$$

be a fixed perverse decomposition associated with $\pi: S \rightarrow C$. We say that the decomposition (2.10) satisfies the condition $(\dagger)$ if the following two properties are satisfied:

(1) The perverse decomposition on $S$ is strongly multiplicative; that is,

$$
G_{i} H^{*}(S, \mathbb{Q}) \cup G_{j} H^{*}(S, \mathbb{Q}) \subset G_{i+j} H^{*}(S, \mathbb{Q}) .
$$




\section{J. SHen AND Z. ZHANG}

(2) The pushforward morphism along the embedding $\Delta_{n}: S \rightarrow S^{n}$ of the small diagonal satisfies

$$
\Delta_{n *}: G_{i} H^{*}(S, \mathbb{Q}) \rightarrow G_{i+2(n-1)} H^{*}\left(S^{n}, \mathbb{Q}\right) .
$$

Proposition 2.8. Suppose that the perverse decomposition (2.10) associated with $\pi: S \rightarrow C$ satisfies the condition $(\dagger)$. Suppose further that

(i) either $S$ is projective and has numerically trivial canonical bundle,

(ii) or $S$ has trivial canonical bundle and admits a nonsingular compactification $\bar{S}$ such that the restriction map $H^{*}(\bar{S}, \mathbb{Q}) \rightarrow H^{*}(S, \mathbb{Q})$ is surjective.

Then the perverse decomposition on $H^{*}\left(S^{[n]}, \mathbb{Q}\right)$ is strongly multiplicative; that is,

$$
G_{i} H^{*}\left(S^{[n]}, \mathbb{Q}\right) \cup G_{j} H^{*}\left(S^{[n]}, \mathbb{Q}\right) \subset G_{i+j} H^{*}\left(S^{[n]}, \mathbb{Q}\right) .
$$

Proof. We closely follow the proof in [Zha17, Section 4]. First of all, a chosen basis of $G_{i} H^{*}(S, \mathbb{Q})$ forms a basis of $H^{*}(S, \mathbb{Q})$. We use this basis to define the abstract perversity on $H^{*}(S, \mathbb{Q})\left\{\mathfrak{S}_{n}\right\}$ as in [Zha17, Definition 4.15]. Then $H^{*}(S, \mathbb{Q})\left\{\mathfrak{S}_{n}\right\}$ is endowed with a canonical perverse decomposition compatible with the one on $H^{*}\left(S^{[n]}, \mathbb{Q}\right)$. Furthermore, by [Zha17, Definitions 4.1 and 4.3], the group $H^{*}(S, \mathbb{Q})^{I}$ has a canonical perverse decomposition for any set $I$.

The strong multiplicativity of $H^{*}(S, \mathbb{Q})$ implies that the strengthened conclusion of [Zha17, Lemma 4.19] holds, namely that the morphism $\varphi^{*}: H^{*}(S, \mathbb{Q})^{I} \rightarrow H^{*}(S, \mathbb{Q})^{J}$ preserves the perverse decompositions; that is, $\varphi^{*} G_{i} H^{*}(S, \mathbb{Q})^{I} \subset G_{i} H^{*}(S, \mathbb{Q})^{J}$. By the condition (ii) on small diagonal embeddings, the conclusion of [Zha17, Lemma 4.20] can be strengthened to the statement that the pushforward $\varphi_{*}: H^{*}(S, \mathbb{Q})^{J} \rightarrow H^{*}(S, \mathbb{Q})^{I}$ increases the perversity by $2(|I|-|J|)$ in the perverse decompositions; that is,

$$
\varphi_{*} G_{i} H^{*}(S, \mathbb{Q})^{J} \subset G_{i+2(n-1)} H^{*}(S, \mathbb{Q})^{I} .
$$

Now the proposition follows from the proofs of [Zha17, Theorems 4.18 and 5.6] together with the strengthened versions of [Zha17, Lemmas 4.19 and 4.20].

\section{Tautological classes on Hilbert schemes}

\subsection{Overview}

We assume that $\pi: S \rightarrow C$ is a fibration from a nonsingular irreducible projective surface to a nonsingular curve such that a general fiber is an elliptic curve. We use the tools developed in Section 2 to analyze the perverse filtration of the Hilbert scheme $S^{[n]}$ associated with the morphism

$$
\pi^{[n]}: S^{[n]} \rightarrow C^{(n)}
$$

and prove Theorem 1.1.

By [LQW02], the tautological classes

$$
\int_{\gamma} c_{k}\left(\mathcal{O}_{Z_{n}}\right) \in H^{*}\left(S^{[n]}, \mathbb{Q}\right), \quad \gamma \in H^{*}(S, \mathbb{Q})
$$

are the generators of the ring $H^{*}\left(S^{[n]}, \mathbb{Q}\right)$. Theorem 1.1 together with Proposition 2.1 calculates the perversity of these generators on the Hilbert scheme $S^{[n]}$.

Since [Zha17] shows that the perverse filtration of $H^{*}\left(S^{[n]}, \mathbb{Q}\right)$ associated with $(3.1)$ is multiplicative when $S$ has numerically trivial canonical bundle, Corollary 1.2 follows from Theorem 1.1. 


\subsection{Exceptional divisors}

In this section, we assume that $\pi: S \rightarrow C$ is a proper surjective morphism from a nonsingular quasi-projective surface to a nonsingular curve $C$.

Let $\partial S^{[n]}$ be the boundary divisor given by the locus of $S^{[n]}$ where the subschemes have length at most $n-1$. For any choice (2.3), we obtain a perverse decomposition

$$
H^{*}\left(S^{[n]}, \mathbb{Q}\right)=\bigoplus_{i \geqslant 0} G_{i} H^{*}\left(S^{[n]}, \mathbb{Q}\right)
$$

by the discussion in Section 2. The following lemma calculates the perversity of the boundary divisor $\partial S^{[n]} \in H^{2}\left(S^{[n]}, \mathbb{Q}\right)$.

Lemma 3.1. For any choice of (2.3), we have

$$
\partial S^{[n]} \in G_{1} H^{2}\left(S^{[n]}, \mathbb{Q}\right) .
$$

In particular, $\mathfrak{p}^{\pi^{[n]}}\left(\partial S^{[n]}\right)=1$.

Proof. Consider the following fibered product Cartesian diagram:

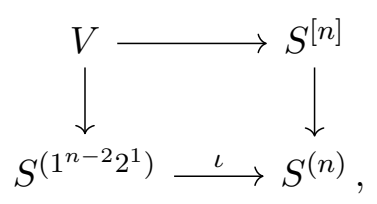

where $\iota=\iota_{\left(1^{n-2} 2^{1}\right)}: S^{\left(1^{n-2} 2^{1}\right)} \rightarrow S^{(n)}$ as in the proof of Proposition 2.5. We have $\left[\partial S^{[n]}\right]=$ $V_{*}\left(\left[S^{\left(1^{n-2} 2^{1}\right)}\right]\right)$, where $V_{*}: H^{*}\left(S^{\left(1^{n-2} 2^{1}\right)}, \mathbb{Q}\right) \rightarrow H^{*}\left(S^{[n]}, \mathbb{Q}\right)$ is viewed as a correspondence. Hence $\partial S^{[n]}$ is identified with the fundamental class $1 \in H^{*}\left(S^{\left(1^{(n-2)} 2^{1}\right)}, \mathbb{Q}\right)[-2]$ in the canonical decomposition (2.4). We conclude the lemma by using the definition of the perverse decomposition for $S^{[n]}$.

We recall that the nested Hilbert scheme is realized as the blow-up of $Z_{n} \subset S^{[n]} \times S$ with the exceptional divisor $E_{n+1}$ given by (2.6). The following relation from [Leh99, Lemma 3.7] expresses $E_{n+1}$ as boundary divisors of Hilbert schemes:

$$
E_{n+1}=\frac{1}{2} \cdot\left(p_{n+1}^{*} \partial S^{[n+1]}-p_{n}^{*} \partial S^{[n]}\right) \in H^{2}\left(S^{[n, n+1]}, \mathbb{Q}\right) .
$$

Here we use the notation as in the diagram (2.5).

For convenience, for any $f: X_{1} \rightarrow X_{2}$, we always denote

$$
f \times \operatorname{id}: X_{1} \times S \rightarrow X_{2} \times S
$$

by $\tilde{f}: X_{1} \times S \rightarrow X_{2} \times S$. The following short exact sequence from [Leh99, Proof of Theorem 4.2, p. 193] compares the universal families of $S^{[n]}$ and $S^{[n+1]}$ :

$$
0 \rightarrow \tilde{\rho}^{*} \mathcal{O}_{\Delta_{S}} \otimes \operatorname{pr}^{*} \mathcal{O}_{S}^{[n, n+1]}\left(-E_{n+1}\right) \rightarrow \tilde{p}_{n+1}^{*} \mathcal{O}_{Z_{n+1}} \rightarrow \tilde{p}_{n}^{*} \mathcal{O}_{Z_{n}} \rightarrow 0
$$

with pr: $S^{[n, n+1]} \times S \rightarrow S^{[n, n+1]}$ the projection and $\Delta_{S} \subset S \times S$ the diagonal.

\subsection{Proof of Theorem 1.1}

We prove Theorem 1.1 by induction on $n$. The induction base is given by the following lemma. Lemma 3.2. Let $\pi: S \rightarrow C$ be a fibration as in Section 3.1, and let $\Delta_{S}$ be the diagonal in $S \times S$. Then

$$
c_{k}\left(\mathcal{O}_{\Delta_{S}}\right) \in P_{k}^{\pi \times \pi} H^{2 k}(S \times S, \mathbb{Q}) .
$$




\section{J. SHen AND Z. ZHANG}

Proof. The cases $k=0,1,4$ are obvious. By [Zha17, Proposition 4.17], the perverse filtration associated with $\pi$ is multiplicative. Hence we can apply [Zha17, Proposition 3.8] to the fundamental class of $S$ and obtain that $\mathfrak{p}^{\pi \times \pi}\left[\Delta_{S}\right] \leqslant 2$. This concludes the $k=2$ case. It remains to show that

$$
c_{3}\left(\mathcal{O}_{\Delta_{S}}\right) \in P_{3}^{\pi \times \pi} H^{6}(S \times S, \mathbb{Q}) .
$$

By the Grothendieck-Riemann-Roch formula, the class $c_{3}\left(\mathcal{O}_{\Delta_{S}}\right)$ is proportional to $\Delta_{*} c_{1}(S)$. Since a general fiber of $\pi: S \rightarrow C$ is an elliptic curve, the class $c_{1}(S)$ is supported on fibers of $\pi$, and therefore $\mathfrak{p}^{\pi}\left(c_{1}(S)\right) \leqslant 1$. Hence (3.5) again follows from [Zha17, Proposition 3.8].

Remark 3.3. The assumption that a general fiber of $\pi: S \rightarrow C$ is an elliptic curve is essential for Theorem 1.1 to hold. For example, if we take $S=\mathbb{P}^{1} \times \mathbb{P}^{1}, C=\mathbb{P}^{1}$, and $\pi: \mathbb{P}^{1} \times \mathbb{P}^{1} \rightarrow \mathbb{P}^{1}$ the natural projection, then the perversity of $c_{3}\left(\mathcal{O}_{\Delta_{S}}\right)$ is 4 . Hence Theorem 1.1 breaks down even when $n=1$.

Since we suppose that the perverse filtration on $S^{[n]}$ associated with $\pi^{[n]}$ is multiplicative, the Künneth decomposition and Proposition 2.1 imply that the perverse filtration on $S^{[n]} \times S$ associated with $\bar{\pi}^{[n]}: S^{[n]} \times S \rightarrow C^{(n)} \times C$ is also multiplicative. Hence Theorem 1.1 is equivalent to $\operatorname{ch}_{k}\left(\mathcal{O}_{Z_{n}}\right) \in P_{k}^{\bar{\pi}^{[n]}} H^{2 k}\left(S^{[n]} \times S, \mathbb{Q}\right)$.

Now we assume that Theorem 1.1 holds for $S^{[n]}$, and we need to prove it for $S^{[n+1]}$. The short exact sequence (3.4) implies that the class $\tilde{p}_{n+1}^{*} \operatorname{ch}_{k}\left(\mathcal{O}_{Z_{n+1}}\right) \in H^{2 k}\left(S^{[n, n+1]}, \mathbb{Q}\right)$ can be expressed as

$$
\tilde{q}_{n}^{*} \operatorname{ch}_{k}\left(\mathcal{O}_{Z_{n}} \otimes \mathcal{O}_{S}\right)+\sum_{k_{1}+k_{2}=k} \tilde{q}_{n}^{*} \operatorname{ch}_{k_{1}}\left(\mathcal{O}_{S[n]} \otimes \mathcal{O}_{\Delta_{S}}\right) \cup \tilde{q}_{n+1}^{*} \operatorname{ch}_{k_{2}}\left(\mathcal{O}_{S^{[n, n+1]}}\left(-E_{n+1}\right) \otimes \mathcal{O}_{S}\right)
$$

In the following, we analyze every term above with respect to the perverse filtrations. First we have

$$
\operatorname{ch}_{k}\left(\mathcal{O}_{Z_{n}} \otimes \mathcal{O}_{S}\right) \in P_{k}^{\bar{\pi}^{[n]}} H^{2 k}\left(S^{[n]} \times S, \mathbb{Q}\right)
$$

due to the induction hypothesis and Proposition 2.1. Also, Lemma 3.2 yields

$$
\operatorname{ch}_{k_{1}}\left(\mathcal{O}_{S^{[n]}} \otimes \mathcal{O}_{\Delta_{S}}\right) \in P_{k_{1}}^{\bar{\pi}^{[n]}} H^{2 k_{1}}\left(S^{[n]} \times S, \mathbb{Q}\right) .
$$

By (3.3), the class of the exceptional divisor $E_{n+1} \in H^{2}\left(S^{[n, n+1]}, \mathbb{Q}\right)$ can be written as

$$
p_{n+1}^{*} \partial S^{[n+1]}-q_{n}^{*}\left(\partial S^{[n]} \times[S]\right) .
$$

Hence we obtain from (3.6), (3.7), (3.8), and Lemma 3.1 that

$$
\tilde{p}_{n+1}^{*} \operatorname{ch}_{k}\left(\mathcal{O}_{Z_{n+1}}\right)=\tilde{q}_{n}^{*} \gamma+\sum_{i} \tilde{p}_{n+1}^{*} \gamma_{i}^{\prime} \cup \tilde{q}_{n}^{*} \gamma_{i}^{\prime \prime}
$$

where

$$
\gamma, \gamma_{i}^{\prime \prime} \in \bigoplus_{l} P_{l}^{\bar{\pi}^{[n]}} H^{2 l}\left(S^{[n]} \times S, \mathbb{Q}\right), \quad \gamma_{i}^{\prime} \in \bigoplus_{l} P_{l}^{\bar{\pi}^{[n+1]}} H^{2 l}\left(S^{[n+1]} \times S, \mathbb{Q}\right) .
$$

Applying $\tilde{p}_{n+1 *}$ to (3.9), we see that the projection formula yields

$$
(n+1) \operatorname{ch}_{k}\left(\mathcal{O}_{Z_{n+1}}\right)=\tilde{p}_{n+1 *}\left(\tilde{q}_{n}^{*} \gamma\right)+\sum_{i} \gamma_{i}^{\prime} \cup \tilde{p}_{n+1 *}\left(\tilde{q}_{n}^{*} \gamma_{i}^{\prime \prime}\right)
$$

Finally, by Corollary 2.6, we have $\tilde{q}_{n}^{*} \alpha \in P_{m}^{\bar{g}_{n}} H^{l}\left(S^{[n, n+1]} \times S, \mathbb{Q}\right)$ for any class $\alpha \in P_{m}^{\bar{\pi}_{n}} H^{l}\left(S^{[n]} \times\right.$ $S, \mathbb{Q})$. Therefore, $\tilde{p}_{n+1 *}\left(\tilde{q}_{n}^{*} \alpha\right) \in P_{m}^{\bar{\pi}_{n+1}} H^{l}\left(S^{[n+1]} \times S, \mathbb{Q}\right)$. In particular, we conclude that the right- 
Perverse filtrations, Hilbert schemes, and $P=W$

hand side of $(3.10)$ lies in $P_{k}^{\bar{\pi}_{n+1}} H^{2 k}\left(S^{[n+1]} \times S, \mathbb{Q}\right)$ due to the multiplicativity of the perverse filtration. We have completed the induction argument.

\section{Parabolic Higgs bundles and the $P=W$ conjecture}

\subsection{Overview}

In this section, we prove the $P=W$ conjecture for the five families of moduli spaces of parabolic Higgs bundles/local systems studied in [Gro14, Theorems 4.1 and 5.1] and [Zha17, Section 5]. Our proof of Theorem 1.4 proceeds along the following steps:

1. We show that there exists a canonical perverse decomposition on the cohomology $H^{*}\left(M_{1}, \mathbb{Q}\right)$ defined by the explicit geometry of the morphism $\pi: M_{1} \rightarrow \mathbb{C}$. We then prove Theorem 1.3 for $n=1$.

2. We deduce that the tautological classes are the ring generators of $H^{*}\left(M_{n}, \mathbb{Q}\right)$. This proves Theorem 1.3(i).

3. The perverse decomposition on $H^{*}\left(M_{1}, \mathbb{Q}\right)$ induces a canonical perverse decomposition on $H^{*}\left(M_{n}, \mathbb{Q}\right)$ for every $n$. Following (a refinement of) the proof of Theorem 0.1 , we locate the tautological classes in the perverse decomposition for $M_{n}$ and complete the proof of Theorem 1.3.

4. We calculate the weights of the tautological classes on the character variety $\mathcal{M}_{n}^{\prime}$. As a consequence, we deduce the decomposition (1.9).

5. Combining the results above, we prove the $P=W$ conjecture for the moduli spaces $M_{n}$ and $\mathcal{M}_{n}^{\prime}$ as a match of the decompositions

$$
\bigoplus_{k, d} G_{k} H^{d}\left(M_{n}, \mathbb{Q}\right) \stackrel{P=W}{=} \bigoplus_{k, d}^{k} \operatorname{Hdg}^{d}\left(\mathcal{M}_{n}^{\prime}\right) .
$$

\subsection{Step 1: 2D moduli spaces and perverse decompositions}

The 2-dimensional moduli spaces associated with the affine Dynkin diagrams $\tilde{A}_{0}, \tilde{D}_{4}, \tilde{E}_{6}, \tilde{E}_{7}$, and $\tilde{E}_{8}$ are constructed in [Gro14, Theorem 1.1]. See also [Zha17, Section 5.1, following Theorem 5.2, pp. 669-670] for a summary. For each Dynkin diagram above, there is a finite group $\Gamma$ acting on an elliptic curve $E$. The induced action on the total cotangent bundle $T^{*} E$ gives a quotient stack $\left[T^{*} E / \Gamma\right]$, and the moduli space $M_{1}$ is given by the crepant resolution of the coarse moduli space of this quotient stack

$$
M_{1} \rightarrow T^{*} E / \Gamma \text {. }
$$

The cohomology groups of $M_{1}$ and their perverse filtrations associated with $\pi_{1}: M_{1} \rightarrow \mathbb{C}$ are described in [Zha17, Sections 5.2 and 5.3].

In the $\tilde{A}_{0}$ case, we have $M_{1} \simeq T^{*} E=E \times \mathbb{C} \stackrel{\pi_{1}}{\longrightarrow} \mathbb{C}$, where $\pi_{1}$ is the projection to the second factor. The perverse filtration associated with $\pi_{1}$ admits a natural splitting

$$
H^{*}\left(M_{1}, \mathbb{Q}\right)=\bigoplus_{i=0}^{2} G_{i} H^{i}\left(T^{*} E, \mathbb{Q}\right)
$$

with $G_{i} H^{i}\left(T^{*} E, \mathbb{Q}\right)=H^{i}\left(T^{*} E, \mathbb{Q}\right)$.

For $\tilde{D}_{4}, \tilde{E}_{6}, \tilde{E}_{7}$, and $\tilde{E}_{8}$, the moduli space $M_{1}$ is nonsingular with trivial canonical bundle. The restriction of the Hitchin fibration $\pi_{1}: M_{1} \rightarrow \mathbb{C}$ to $\mathbb{C}^{*}=\mathbb{C} \backslash\{0\}$ is an isotrivial fibration 


\section{J. SHen AND Z. ZHANG}

with each closed fiber isomorphic to the elliptic curve $E$, that is, $\pi_{1}: \pi_{1}^{-1}\left(\mathbb{C}^{*}\right) \rightarrow \mathbb{C}^{*} \subset \mathbb{C}$, while the closed fiber over $0 \in \mathbb{C}$ has the dual graph given by the corresponding affine Dynkin diagram. All the nontrivial cohomology groups of $M_{1}$ are

$$
H^{0}\left(M_{1}, \mathbb{Q}\right)=\left\langle\left[M_{1}\right]\right\rangle, \quad H^{2}\left(M_{1}, \mathbb{Q}\right)=\left\langle[s],\left[E_{1}\right], \ldots,\left[E_{K}\right]\right\rangle .
$$

Here $[s]$ is class of a section of $\pi_{1}$, the $\left[E_{i}\right]$ are the exceptional divisors of the resolution (4.1), and

$$
K= \begin{cases}4, & \tilde{D}_{4} \text { case } \\ 6, & \tilde{E}_{6} \text { case } \\ 7, & \tilde{E}_{7} \text { case } \\ 8, & \tilde{E}_{8} \text { case }\end{cases}
$$

We define the following perverse decomposition associated with $\pi_{1}$ :

$$
G_{0} H^{0}\left(M_{1}, \mathbb{Q}\right)=\left\langle\left[M_{1}\right]\right\rangle, \quad G_{1} H^{2}\left(M_{1}, \mathbb{Q}\right)=\left\langle\left[E_{1}\right], \ldots,\left[E_{K}\right]\right\rangle, \quad G_{2} H^{2}\left(M_{1}, \mathbb{Q}\right)=\langle[s]\rangle .
$$

In the $\tilde{A}_{0}$ case, the group $\Gamma$ is trivial, and $\mathbb{P}_{\Gamma}=E=\mathcal{I} E$.

In the $\tilde{D}_{4}, \tilde{E}_{6}, \tilde{E}_{7}$, and $\tilde{E}_{8}$ cases, the (ungraded) cohomology groups of the inertia stacks $\mathcal{I} \mathbb{P}_{\Gamma}$, $\mathcal{I}\left[T^{*} E / \Gamma\right]$, and the moduli space $M_{1}$ can be canonically identified,

$$
H_{\text {orb }}^{*}\left(\mathbb{P}_{\Gamma}, \mathbb{Q}\right)=H^{*}\left(\mathcal{I}\left[T^{*} E / \Gamma\right], \mathbb{Q}\right)=H^{*}\left(M_{1}, \mathbb{Q}\right) .
$$

The first equality is induced by the projection $\mathcal{I}\left[T^{*} E / \Gamma\right] \rightarrow \mathcal{I} \mathbb{P}_{\Gamma}$, and the second equality is given by the McKay correspondence. ${ }^{3}$ By definition, we have

$$
H_{\mathrm{orb}}^{*}\left(\mathbb{P}_{\Gamma}, \mathbb{Q}\right)=H^{*}\left(\mathbb{P}_{\Gamma}, \mathbb{Q}\right) \oplus \bigoplus_{i=1}^{K} \mathbb{Q}\left[e_{i}\right],
$$

where every $\left[e_{i}\right] \in H_{\text {orb }}^{0}\left(\mathbb{P}_{\Gamma}, \mathbb{Q}\right)$ is given by an element of the isotropy group of a $\Gamma$-fixed point corresponding to the exceptional divisor $E_{i} \in H^{2}\left(M_{1}, \mathbb{Q}\right)$ via $(4.3)$. Assume that $\left(\mathcal{E}_{1}, \sigma_{1}\right)$ is the universal family on $M_{1} \times \mathcal{I} \mathbb{P}_{\Gamma}$ obtained as the pullback of the universal Higgs bundle on $M_{1} \times \mathbb{P}_{\Gamma}$ induced by the Poincaré line bundle (4.4). We first prove Theorem 1.3 in the $n=1$ case.

Proposition 4.1. Theorem 1.3 holds for $n=1$.

Proof. We first treat the $\tilde{A}_{0}$ case. The universal line bundle $\mathcal{E}_{1}$ on $T^{*} E \times E$ is the pullback of the Poincaré line bundle

$$
\mathcal{P}=\mathcal{O}_{E \times E}\left(\Delta-0_{E} \times E-E \times 0_{E}\right)
$$

from the projection $M_{1} \times E \rightarrow E \times E$, where $0_{E}$ denotes the origin of the elliptic curve. Hence the Künneth factor of $c_{0}(\mathcal{P})$ on $M_{1}$ is the fundamental class $\left[M_{1}\right] \in G_{0} H^{0}\left(M_{1}, \mathbb{Q}\right)$, and the Künneth factors of $c_{1}(\mathcal{P})$ are the odd-degree classes in $G_{1} H^{1}\left(M_{1}, \mathbb{Q}\right)$. They all lie in the correct pieces of the decomposition $G_{\bullet}$ and generate the total cohomology $H^{*}\left(M_{1}, \mathbb{Q}\right)$.

Now we consider the $\tilde{D}_{4}, \tilde{E}_{6}, \tilde{E}_{7}$, and $\tilde{E}_{8}$ cases, where the $\Gamma$-group actions are nontrivial. We view $\operatorname{ch}\left(\mathcal{E}_{1}\right)$ as a correspondence on the cohomology:

$$
\operatorname{ch}\left(\mathcal{E}_{1}\right): H^{*}\left(\mathcal{I P}_{\Gamma}, \mathbb{Q}\right) \rightarrow H^{*}\left(M_{1}, \mathbb{Q}\right) .
$$

By the construction of [Gro14], the correspondence (4.5) can be factorized as

$$
H^{*}\left(\mathcal{I} \mathbb{P}_{\Gamma}, \mathbb{Q}\right) \stackrel{\Phi_{1}}{\longrightarrow} H^{*}\left(\mathcal{I}\left[T^{*} E / \Gamma\right], \mathbb{Q}\right) \stackrel{\Phi_{2}}{\longrightarrow} H^{*}\left(M_{1}, \mathbb{Q}\right)
$$

\footnotetext{
${ }^{3}$ In this case, we can also view the second equality as an identification in the $K$-theory with $\mathbb{Q}$-coefficients.
} 


\section{Perverse filtrations, Hilbert schemes, and $P=W$}

Here $\Phi_{1}$ is given by the $\Gamma$-equivariant Fourier-Mukai functor induced by the $\Gamma$-equivariant Poincaré line bundle $\mathcal{P} \in D^{b}\left(E \times T^{*} E\right)$, and $\Phi_{2}$ is an isomorphism given by the McKay correspondence. In particular, we obtain that (4.5) is an isomorphism. This implies that the tautological classes generate $H^{*}\left(M_{1}, \mathbb{Q}\right)$ and proves the first part of Theorem 1.3.

For part (ii), a direct calculation using the factorization (4.6) gives the following equations:

$$
\begin{aligned}
\Phi_{2} \circ \Phi_{1}\left(\left[\mathrm{pt} \in \mathbb{P}_{\Gamma}\right]\right) & =\left[M_{1}\right] \in G_{0} H^{0}\left(M_{1}, \mathbb{Q}\right), \\
\Phi_{2} \circ \Phi_{1}\left\langle\left[e_{1}\right],\left[e_{2}\right], \ldots,\left[e_{K}\right]\right\rangle & =\left\langle\left[E_{1}\right],\left[E_{2}\right], \ldots,\left[E_{K}\right]\right\rangle=G_{1} H^{2}\left(M_{1}, \mathbb{Q}\right), \\
\Phi_{2} \circ \Phi_{1}\left(\left[\mathbb{P}_{\Gamma}\right]\right) & =[s] \in G_{2} H^{2}\left(M_{1}, \mathbb{Q}\right) .
\end{aligned}
$$

By looking at the cohomological degrees, we see that the three identities above are induced by the factors $\operatorname{ch}_{0}\left(\mathcal{E}_{1}\right), \operatorname{ch}_{1}\left(\mathcal{E}_{1}\right)$, and $\operatorname{ch}_{2}\left(\mathcal{E}_{1}\right)$, respectively. In conclusion, the Künneth factors of $\operatorname{ch}_{k}\left(\mathcal{E}_{1}\right)$ on $M_{1}$ lie in $G_{k} H^{*}\left(M_{1}, \mathbb{Q}\right)$. This completes the proof of the proposition in the $\tilde{D}_{4}, \tilde{E}_{6}, \tilde{E}_{7}$, and $\tilde{E}_{8}$ cases.

\subsection{Step 2: Tautological classes on $M_{n}$}

We show in this section that the tautological classes

$$
\int_{\alpha} c_{k}(\mathcal{E}) \in H^{*}\left(M_{n}, \mathbb{Q}\right), \quad \alpha \in H_{\text {orb }}^{*}\left(\mathbb{P}_{\Gamma}, \mathbb{Q}\right)
$$

generate the cohomology ring $H^{*}\left(M_{n}, \mathbb{Q}\right)$. It is parallel to a result of Markman [Mar02] in the case of (nonparabolic) Higgs bundles on a compact Riemann surface of genus at least 2 . See [HT04, HT03] for more concrete descriptions in the rank 2 case. However, our proof relies on the structure for the cohomology of the Hilbert scheme of points on a projective surface [LQW02], which is different from the approach in [Mar02].

Proof of Theorem 1.3(i). (1) The $\tilde{A}_{0}$ case. We first treat the $\tilde{A}_{0}$ case. For a projective nonsingular surface $S$, recall that the result of Li-Qin-Wang [LQW02] implies that the image of the correspondence

$$
\operatorname{ch}\left(\mathcal{O}_{Z_{n}}\right): H^{*}(S, \mathbb{Q}) \rightarrow H^{*}\left(S^{[n]}, \mathbb{Q}\right)
$$

generates the $\operatorname{ring} H^{*}\left(S^{[n]}, \mathbb{Q}\right)$, where $Z_{n}$ is the universal subscheme. For our purpose, we take a compactification of $M_{1}$, namely $M_{1}=T^{*} E \subset E \times \mathbb{P}^{1}=\bar{M}_{1}$, which induces a natural compactification $\bar{M}_{n}$ of $M_{n}$ by taking the Hilbert scheme $\bar{M}_{1}^{[n]}$ of $n$ points of the nonsingular surface $\bar{M}_{1}$, that is, $M_{n} \subset \bar{M}_{n}:=\bar{M}_{1}^{[n]}$. The restriction map on the cohomology groups

$$
H^{*}\left(\bar{M}_{n}, \mathbb{Q}\right) \stackrel{\text { res. }}{\longrightarrow} H^{*}\left(M_{n}, \mathbb{Q}\right)
$$

is surjective by the decomposition (2.4). We define $\Xi_{n}^{\prime} \subset \bar{M}_{n} \times \bar{M}_{1}$ to be the universal subscheme and let $\Xi_{n}$ be its restriction on $M_{n} \times \bar{M}_{1}$.

By the construction of [Gro14], the derived functor $D^{b}(E) \rightarrow D^{b}\left(M_{n}\right)$ given by the universal family $\mathcal{E}_{n} \in D^{b}\left(M_{n} \times E\right)$ can be factorized as

$$
D^{b}(E) \rightarrow D^{b}\left(M_{1}\right) \rightarrow D^{b}\left(M_{n}\right)
$$

where the first morphism is induced by the universal family $\mathcal{E}_{1}$ on $M_{1} \times E$ and the second morphism is induced by the structure sheaf of the universal subscheme in $M_{n} \times M_{1}=M_{1}^{[n]} \times M_{1} \cdot{ }^{4}$

${ }^{4}$ Although $M_{1}$ is not proper, the Fourier-Mukai transform $D^{b}\left(M_{1}\right) \rightarrow D^{b}\left(M_{n}\right)$ is well defined. This is because the support of $\mathcal{O}_{\Xi_{n}}$ in $M_{n} \times M_{1}$ is proper over $M_{n}$. 


\section{J. SHen AND Z. ZHANG}

We consider the following commutative diagram of correspondences for cohomology groups,

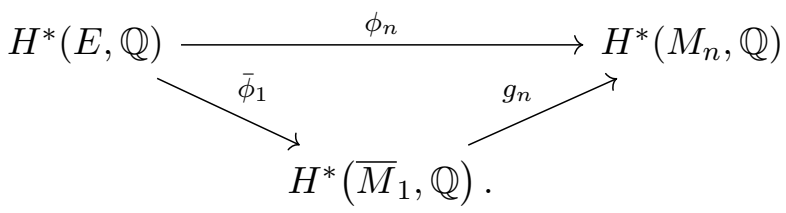

Here $\phi_{n}$ is induced by the functor (4.9), $\bar{\phi}_{1}$ is induced by the pullback of the Poincare line bundle on $E \times E$ via the natural projection $\bar{M}_{1} \rightarrow E \times E$, and $g_{n}$ is induced by $\mathcal{O}_{\Xi_{n}} \in \operatorname{Coh}\left(M_{n} \times \bar{M}_{1}\right)$ (the diagram is commutative since the Todd class of $\bar{M}_{1}$ is supported on the divisor $\bar{M}_{1} \backslash M_{1}$ ). For the proof of Theorem 1.3(i), it suffices to show that the image of $\phi_{n}$ generates the cohomology ring $H^{*}\left(M_{n}, \mathbb{Q}\right)$.

By [LQW02] and the surjectivity of the restriction map (4.8), we observe that the image of $g_{n}$ generates $H^{*}\left(M_{n}, \mathbb{Q}\right)$. Moreover, a direct calculation implies that any class in the image of $\bar{\phi}_{1}$ does not intersect the divisor $E \times\{\infty\}=\bar{M}_{1} \backslash M_{1}$. Since the support of the class $\operatorname{ch}\left(\mathcal{O}_{\Xi_{n}}\right)$ does not intersect the locus $\left(\bar{M}_{1} \backslash M_{1}\right) \times M_{n}$, we obtain from the projective bundle formula that

$$
H^{*}\left(\bar{M}_{1}, \mathbb{Q}\right)=H^{*}\left(M_{1}\right) \oplus N=\operatorname{Im}\left(\bar{\phi}_{1}\right) \oplus N,
$$

where $N$ consists of the classes supported on $\bar{M}_{1} \backslash M_{1}$, and therefore $N \subset \operatorname{Ker}\left(g_{n}\right)$. As a consequence, we have $\operatorname{Im}\left(\phi_{n}\right)=\operatorname{Im}\left(g_{n}\right)$. This completes the proof in the $\tilde{A}_{0}$ case. (2) The other cases. The cases of $\tilde{D}_{4}, \tilde{E}_{6}, \tilde{E}_{7}$, and $\tilde{E}_{8}$ are similar. The action of $\Gamma$ on an elliptic curve $E$ can be lifted to the total space of the cotangent bundle $T^{*} E$. We consider the induced $\Gamma$-action on the surface $E \times \mathbb{P}^{1}$ (viewed as a compactification of the open surface $T^{*} E$ ). Since $M_{1}$ is the crepant resolution of the singular surface $T^{*} E / \Gamma$, a nonsingular resolution of the quotient $E \times \mathbb{P}^{1} / \Gamma$, denoted by $\bar{M}_{1}$, is then a compactification of $M_{1}$ with the surjective restriction map

$$
H^{*}\left(\bar{M}_{1}, \mathbb{Q}\right) \stackrel{\text { res. }}{\longrightarrow} H^{*}\left(M_{1}, \mathbb{Q}\right) .
$$

Geometrically, $\bar{M}_{1}$ contains $M_{1}$ as an open sub-surface, and the boundary is $\bar{M}_{1, \infty}=\cup_{i}^{l} D_{i} \cup F$, where the $D_{i}$ are the exceptional curves. Hence we have

$$
H^{*}\left(\bar{M}_{1}, \mathbb{Q}\right)=H^{*}\left(M_{1}, \mathbb{Q}\right) \oplus N, \quad N=\left\langle\left[D_{1}\right], \ldots,\left[D_{l}\right],[F],[\mathrm{pt}]\right\rangle .
$$

As in the $\tilde{A}_{0}$ case, we consider the Hilbert scheme $\bar{M}_{n}=\bar{M}_{1}^{[n]}$ as a nonsingular compactification of $M_{n}$. By the surjectivity of (4.10) and the decomposition (2.4), we obtain that the restriction map res. : $H^{*}\left(\bar{M}_{n}, \mathbb{Q}\right) \rightarrow H^{*}\left(M_{n}, \mathbb{Q}\right)$ is also surjective. Since $M_{n}$ is the moduli space of $\Gamma$-equivariant stable parabolic Higgs bundles on the elliptic curve $E$, see [Gro14], the derived functor $D^{b}([E / \Gamma]) \rightarrow D^{b}\left(M_{n}\right)$ induced by the universal family can be factorized as

$$
D^{b}([E / \Gamma]) \rightarrow D^{b}\left(M_{1}\right) \rightarrow D^{b}\left(M_{n}\right),
$$

as in (4.9).

On the level of cohomology, we consider the following commutative diagram of correspondences:

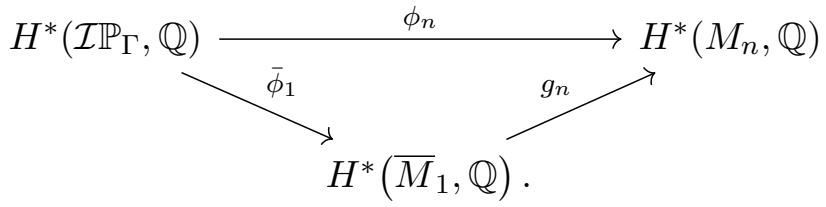

Here $\phi_{n}$ is induced by the pullback of the universal family on $M_{n} \times[E / \Gamma]$ to $M_{n} \times \mathcal{I P}_{\Gamma}$, the 
morphism

$$
\bar{\phi}_{1}: H^{*}\left(\mathcal{I} \mathbb{P}_{\Gamma}, \mathbb{Q}\right) \rightarrow H^{*}\left(\mathcal{I}\left[T^{*} E / \Gamma\right], \mathbb{Q}\right)=H^{*}\left(M_{1}, \mathbb{Q}\right) \hookrightarrow H^{*}\left(\bar{M}_{1}, \mathbb{Q}\right)
$$

is induced by the $\Gamma$-equivariant universal Poincaré sheaf on $E \times T^{*} E$ (see Proposition 4.1), and $g_{n}$ is induced by the structure sheaf $\mathcal{O}_{\Xi_{n}}$.

We obtain from (4.11) that

$$
H^{*}\left(\bar{M}_{1}, \mathbb{Q}\right)=H^{*}\left(M_{1}, \mathbb{Q}\right) \oplus N=\operatorname{Im}\left(\bar{\phi}_{1}\right) \oplus N,
$$

where $N$ is generated by the classes supported on $\bar{M}_{1} \backslash M_{1}$. The same proof as in the $\tilde{A}_{0}$ case implies that $N \subset \operatorname{Ker}\left(g_{n}\right)$. Hence $\operatorname{Im}\left(\phi_{n}\right)=\operatorname{Im}\left(g_{n}\right)$. In particular, we conclude that the image of $\phi_{n}$ generates $H^{*}\left(M_{n}, \mathbb{Q}\right)$.

\subsection{Step 3: Perverse decompositions and tautological classes}

By Section 3, the decomposition

$$
H^{*}\left(M_{1}, \mathbb{Q}\right)=\bigoplus_{i=0}^{2} G_{i} H^{*}\left(M_{1}, \mathbb{Q}\right)
$$

of Section 4.2 induces the perverse decomposition (1.6) for every moduli space $M_{n}=M_{1}^{[n]}$. The purpose of this section is to calculate the location of the tautological classes (4.7) in the perverse decomposition of $H^{*}\left(M_{n}, \mathbb{Q}\right)$ and complete the proof of Theorem 1.3.

Lemma 4.2. The perverse decomposition $G_{\bullet} H^{*}\left(M_{n}, \mathbb{Q}\right)$ is strongly multiplicative; that is,

$$
G_{i} H^{*}\left(M_{n}, \mathbb{Q}\right) \cup G_{j} H^{*}\left(M_{n}, \mathbb{Q}\right) \subset G_{i+j} H^{*}\left(M_{n}, \mathbb{Q}\right) .
$$

Proof. It suffices to check that the conditions of Proposition 2.8 hold for the surface $M_{1}$. The conditions $(\dagger)(1)$ and (ii) are clearly satisfied, and $(\dagger)(2)$ follows from [Zha17, Proposition 3.8].

Let $\pi^{\prime}: \bar{M}_{1} \rightarrow \mathbb{P}^{1}$ be the natural projection which compactifies the Hitchin fibration $\pi_{1}: M_{1} \rightarrow \mathbb{C}$. By the description (4.11), the perverse filtration associated with $\pi^{\prime}$ admits a canonical splitting

$$
H^{*}\left(\bar{M}_{1}, \mathbb{Q}\right)=\bigoplus_{i=0}^{2} G_{i} H^{*}\left(\bar{M}_{1}, \mathbb{Q}\right)
$$

satisfying that $[\mathrm{pt}] \in G_{2} H^{4}\left(\bar{M}_{1}, \mathbb{Q}\right),[F] \in G_{0} H^{2}\left(\bar{M}_{1}, \mathbb{Q}\right)$, and $\left[D_{i}\right] \in G_{1} H^{2}\left(\bar{M}_{1}, \mathbb{Q}\right)$, and its restriction to $H^{*}\left(M_{1}, \mathbb{Q}\right)$ coincides with $(4.13)$. (We omit the discussion for the $\widetilde{A}_{0}$ case since the decomposition (4.14) is obviously obtained for $\pi^{\prime}: \bar{M}_{1}=E \times \mathbb{P}^{1} \rightarrow \mathbb{P}^{1}$.)

For notational convenience, if the cohomology of each variety $X_{i}$ admits a perverse decomposition $G_{\bullet} H^{*}\left(X_{i}, \mathbb{Q}\right)$ with respect to a morphism $X_{i} \rightarrow Y_{i}$, we define

$$
G_{k} H^{*}\left(X_{1} \times \cdots \times X_{m}, \mathbb{Q}\right)=\left\langle\alpha_{1} \otimes \cdots \otimes \alpha_{m} ; \alpha_{i} \in G_{k_{i}} H^{*}\left(X_{i}, \mathbb{Q}\right), \sum_{i} k_{i}=m\right\rangle,
$$

which gives a perverse decomposition for $X_{1} \times X_{2} \times \cdots \times X_{m}$ with respect to the Cartesian product

$$
X_{1} \times X_{2} \times \cdots \times X_{m} \rightarrow Y_{1} \times Y_{2} \times \cdots \times Y_{m}
$$

by Proposition 2.1. In particular, (4.14) induces a perverse decomposition for $M_{n} \times \bar{M}_{1}$ with respect to the morphism $\pi_{n} \times \pi^{\prime}: M_{n} \times \bar{M}_{1} \rightarrow \mathbb{C}^{n} \times \mathbb{P}^{1}$.

Recall that $\Xi_{n}$ is the restricted universal subscheme of $M_{n} \times \bar{M}_{1}$. The following theorem is parallel to Theorem 1.1. We show it by the induction scheme of Section 3.3. 


\section{J. SHen AND Z. ZHANG}

Theorem 4.3. We have

$$
\operatorname{ch}_{k}\left(\mathcal{O}_{\Xi_{n}}\right) \in G_{k} H^{2 k}\left(M_{n} \times \bar{M}_{1}, \mathbb{Q}\right) .
$$

Proof. We first verify the theorem for $n=1$. The subscheme $Z_{1} \subset M_{1} \times \bar{M}_{1}$ is the restriction of the diagonal $\Delta \subset \bar{M}_{1} \times \bar{M}_{1}$. By a general calculation of the perversity of the diagonal class [Zha17, Proposition 3.8], we have

$$
[\Delta] \in G_{2} H^{4}\left(\bar{M}_{1} \times \bar{M}_{1}, \mathbb{Q}\right) .
$$

Hence we obtain via the restriction map (4.10) that $c_{2}\left(\mathcal{O}_{Z_{1}}\right) \in G_{2} H^{4}\left(M_{1} \times \bar{M}_{1}, \mathbb{Q}\right)$. Now since $c_{1}\left(\mathcal{O}_{Z_{1}}\right)=c_{4}\left(\mathcal{O}_{Z_{1}}\right)=0$, it suffices to show that $c_{3}\left(\mathcal{O}_{Z_{1}}\right) \in G_{3} H^{6}\left(M_{1} \times \bar{M}_{1}, \mathbb{Q}\right)$.

In fact, by the Grothendieck-Riemann-Roch formula, the class $c_{3}\left(\mathcal{O}_{\Delta}\right)$ is proportional to the class $\operatorname{pr}_{1}^{*} c_{1}\left(\bar{M}_{1}\right) \cup[\Delta] \in H^{6}\left(\bar{M}_{1} \times \bar{M}_{1}, \mathbb{Q}\right)$. Its restriction to $M_{1} \times \bar{M}_{1}$ vanishes due to the fact that $c_{1}\left(\bar{M}_{1}\right)$ is supported on $\bar{M}_{1} \backslash M_{1}$.

The rest of the proof is nearly the same as the induction argument in Section 3.3. The only minor difference is that, instead of considering the perverse filtration $P_{\bullet} H^{*}\left(M_{n} \times \bar{M}_{1}\right)$, we work with the perverse decomposition $G_{\bullet} H^{*}\left(M_{n} \times \bar{M}_{1}\right)$. The corresponding comparison results for the pushforward morphism along $M_{1}^{[n, n+1]} \rightarrow M_{n+1}$ and the pullback morphism along $M_{1}^{[n, n+1]} \rightarrow$ $M_{n} \times M_{1}$ are given by Propositions 2.4 and 2.5. The precise location of the exceptional divisor in the decomposition $G_{\bullet} H^{*}\left(M_{n}, \mathbb{Q}\right)$ is calculated in Lemma 3.1. Finally, the multiplicativity of the perverse filtration used in Section 3.3 is replaced by the strong multiplicativity for the decomposition $G_{\bullet} H^{*}\left(M_{n}, \mathbb{Q}\right)$ (see Lemma 4.2).

For convenience, we introduce the trivial perverse decomposition on the inertia stack $\mathcal{I} \mathbb{P}_{\Gamma}$,

$$
G_{0} H^{*}\left(\mathcal{I P}_{\Gamma}, \mathbb{Q}\right)=H^{*}\left(\mathcal{I} \mathbb{P}_{\Gamma} \cdot \mathbb{Q}\right)
$$

By the Künneth decomposition, it induces the perverse decompositions $G_{\bullet} H^{*}\left(M_{n} \times \mathcal{I} \mathbb{P}_{\Gamma}, \mathbb{Q}\right)$ and $G_{\bullet} H^{*}\left(M_{n} \times \bar{M}_{1} \times \mathcal{I P}_{\Gamma}, \mathbb{Q}\right)$ which split the perverse filtrations associated with the morphisms

$$
\pi_{n} \times \mathrm{id}: M_{n} \times \mathcal{I P}_{\Gamma} \rightarrow \mathbb{C}^{n} \times \mathcal{I} \mathbb{P}_{\Gamma}
$$

and

$$
\pi_{n} \times \pi^{\prime} \times \mathrm{id}: M_{n} \times \bar{M}_{1} \times \mathcal{I} \mathbb{P}_{\Gamma} \rightarrow \mathbb{C}^{n} \times \mathbb{P}^{1} \times \mathcal{I} \mathbb{P}_{\Gamma}
$$

We define the class on the product $M_{n} \times \bar{M}_{1} \times \mathcal{I} \mathbb{P}_{\Gamma}$ :

$$
I(\alpha, \gamma)=\int_{\gamma} \operatorname{pr}_{12}^{*} \operatorname{ch}\left(\mathcal{O}_{\Xi_{n}}\right) \cup \operatorname{pr}_{23}^{*} \alpha \in H^{*}\left(M_{n} \times \mathcal{I} \mathbb{P}_{\Gamma}, \mathbb{Q}\right)
$$

with $\gamma \in H^{*}\left(\bar{M}_{1}, \mathbb{Q}\right), \alpha \in H^{*}\left(\bar{M}_{1} \times \mathcal{I} \mathbb{P}_{\Gamma}, \mathbb{Q}\right)$, and $\operatorname{pr}_{i j}$ the projections. Since the Chern character $\operatorname{ch}\left(\mathcal{O}_{\Xi_{n}}\right)$ is supported on the open subset $M_{n} \times M_{1} \subset M_{n} \times \bar{M}_{1}$, the class $I(\alpha, \gamma)$ only depends on the restriction of $\alpha$ to $H^{*}\left(M_{1} \times \mathcal{I} \mathbb{P}_{\Gamma}, \mathbb{Q}\right)$ by the Künneth decomposition. Hence $I(\alpha, \gamma)$ is well defined for $\alpha \in H^{*}\left(M_{1} \times \mathcal{I} \mathbb{P}_{\Gamma}, \mathbb{Q}\right)$.

Let $\left(\mathcal{E}_{n}, \theta_{n}\right)$ denote the universal family on $M_{n} \times \mathbb{P}_{\Gamma}$. For our purpose, it suffices to show that ${ }^{5}$

$$
\operatorname{ch}\left(\mathcal{E}_{n}\right) \in \bigoplus_{i} G_{i} H^{2 i}\left(M_{n} \times \mathcal{I P}_{\Gamma}, \mathbb{Q}\right)
$$

\footnotetext{
${ }^{5}$ For notational convenience, we also use $\operatorname{ch}\left(\mathcal{E}_{n}\right)$ to denote the pullback of its corresponding class on $M_{n} \times \mathbb{P}_{\Gamma}$ to $M_{n} \times \mathcal{I} \mathbb{P}_{\Gamma}$.
} 


\section{Perverse filtrations, Hilbert schemes, and $P=W$}

Since the derived functor induced by the universal family $\mathcal{E}_{n}$ on $M_{n} \times \mathbb{P}_{\Gamma}$ is factorized as (4.12), we obtain that (4.16) is equivalent to the following condition:

$$
I\left(\operatorname{ch}\left(\mathcal{E}_{1}\right),\left[\bar{M}_{1}\right]\right) \in \bigoplus_{i} G_{i} H^{2 i}\left(M_{n} \times \mathcal{I} \mathbb{P}_{\Gamma}, \mathbb{Q}\right) .
$$

In the following, we prove Theorem 1.3(ii) by verifying (4.17).

Proof of Theorem 1.3(ii). Assume that a variety $X$ admits a perverse decomposition $G \bullet H^{*}(X, \mathbb{Q})$. We call a class $\alpha \in H^{*}(X, \mathbb{Q})$ balanced if

$$
\alpha \in \bigoplus_{i} G_{i} H^{2 i}(X, \mathbb{Q}) .
$$

By the calculation in the proof of Proposition 4.1, the class $\operatorname{ch}\left(\mathcal{E}_{1}\right) \in H^{*}\left(M_{1} \times \mathcal{I} \mathbb{P}_{\Gamma}, \mathbb{Q}\right)$ is balanced. The Chern character $\operatorname{ch}\left(\mathcal{O}_{\Xi_{n}}\right)$ is also balanced by Theorem 4.3.

Since $\left[M_{n}\right] \in G_{0} H^{0}\left(M_{n}, \mathbb{Q}\right)$ and $\left[\mathcal{I} \mathbb{P}_{\Gamma}\right] \in G_{0} H^{0}\left(\mathcal{I} \mathbb{P}_{\Gamma}, \mathbb{Q}\right)$, Proposition 2.1 implies that the pullbacks $\mathrm{pr}_{12}^{*}$ and $\mathrm{pr}_{23}^{*}$ preserve the perverse decompositions. Hence we deduce from Lemma 4.2 that the class $\operatorname{pr}_{12}^{*} \operatorname{ch}\left(\mathcal{O}_{\Xi_{n}}\right) \cup \operatorname{pr}_{23}^{*} \operatorname{ch}\left(\mathcal{E}_{1}\right)$ is balanced. ${ }^{6}$ Note that the integration over the fundamental class $\left[\bar{M}_{1}\right]$ is equivalent to picking up the Künneth factor corresponding to the point class $[\mathrm{pt}] \in G_{2} H^{4}\left(\bar{M}_{1}, \mathbb{Q}\right)$. We find that $I\left(\operatorname{ch}\left(\mathcal{E}_{1}\right),\left[\bar{M}_{1}\right]\right)$ is balanced.

\subsection{Step 4: Weight filtrations and tautological classes}

The tautological classes for the character varieties are studied in [HR08, She17]. In this section we compute the weights of the tautological classes following the method in [She17]. Let $\mathcal{M}_{n}^{\prime}$ denote the parabolic character variety corresponding to the parabolic Higgs moduli $M_{n}$ via the nonabelian Hodge theorem; see [GNR01, Section 5.1], [Gro14, Theorem 5.1], and [Zha17, Section 5.1] for details. By the parabolic nonabelian Hodge theorem [BGM20, Theorem 7.10], the character variety $\mathcal{M}_{n}^{\prime}$ is canonically homeomorphic to $M_{n}$. The following result is parallel to Theorem 1.3 for Higgs bundles.

Theorem 4.4. Let $\mathcal{E}_{n}^{\prime}$ be the universal family on $\mathcal{M}_{n}^{\prime} \times \mathcal{I P}_{\Gamma}$ which is obtained as the pullback of the universal family on $\mathcal{M}_{n}^{\prime} \times \mathbb{P}_{\Gamma}$ (given by the flat bundles corresponding to the local systems via the Riemann-Hilbert correspondence). The following statements hold:

(i) The tautological classes

$$
\int_{\alpha} \mathrm{c}_{k}\left(\mathcal{E}_{n}^{\prime}\right) \in H^{*}\left(\mathcal{M}_{n}^{\prime}, \mathbb{Q}\right), \quad \alpha \in H_{\text {orb }}^{*}\left(\mathbb{P}_{\Gamma}, \mathbb{Q}\right)
$$

generate the cohomology ring $H^{*}\left(\mathcal{M}_{n}^{\prime}, \mathbb{Q}\right)$.

(ii) We have

$$
\int_{\alpha} \mathrm{c}_{k}\left(\mathcal{E}_{n}^{\prime}\right) \in{ }^{k} \operatorname{Hdg}^{*}\left(\mathcal{M}_{n}^{\prime}, \mathbb{Q}\right) \quad \text { for any } \alpha \in H_{\text {orb }}^{*}\left(\mathbb{P}_{\Gamma}, \mathbb{Q}\right)
$$

Proof. Recall from [Gro14] that the moduli space $M_{n}$ parametrizes all triples $(\mathcal{F}, \theta, v)$, where $(\mathcal{F}, \theta)$ is a semistable degree 0 Higgs bundle on the orbifold $\mathbb{P}_{\Gamma}$ and $v$ is a vector in the fiber $\mathcal{F}_{0}$. Here the stability condition refers to that any Higgs sub-bundle containing $v$ has negative degree.

${ }^{6}$ We view $\operatorname{ch}\left(\mathcal{E}_{1}\right) \in H^{*}\left(M_{1} \times \mathcal{I P}_{\Gamma}, \mathbb{Q}\right)$ as a class in $H^{*}\left(\bar{M}_{1} \times \mathcal{I} \mathbb{P}_{\Gamma}, \mathbb{Q}\right)$ by the Künneth decomposition and $H^{*}\left(\bar{M}_{1}, \mathbb{Q}\right)=H^{*}\left(M_{1}, \mathbb{Q}\right) \oplus N$. 


\section{J. SHen AND Z. ZHANG}

By forgetting parabolic structures of the objects parametrized by $M_{n}$, we obtain a morphism $M_{n} \rightarrow \mathcal{H} i g g s_{n}\left(\mathbb{P}_{\Gamma}\right)$, where $\mathcal{H}$ iggs $s_{n}\left(\mathbb{P}_{\Gamma}\right)$ denotes the moduli stack of degree 0 rank $n$ Higgs bundles on $\mathbb{P}_{\Gamma}$ or, equivalently, the moduli stack of $\Gamma$-equivariant Higgs bundles on $E$ of degree 0 and rank $n$.

Similarly, we denote by $\operatorname{Loc}\left(\mathbb{P}_{\Gamma}\right)$ the moduli stack of $\Gamma$-equivariant rank $n$ local systems on $E$. Let $\Delta_{\mathcal{I}}$ be a simplicial resolution of the inertia stack $\mathcal{I} \mathbb{P}_{\Gamma}$. We have the following commutative diagrams:

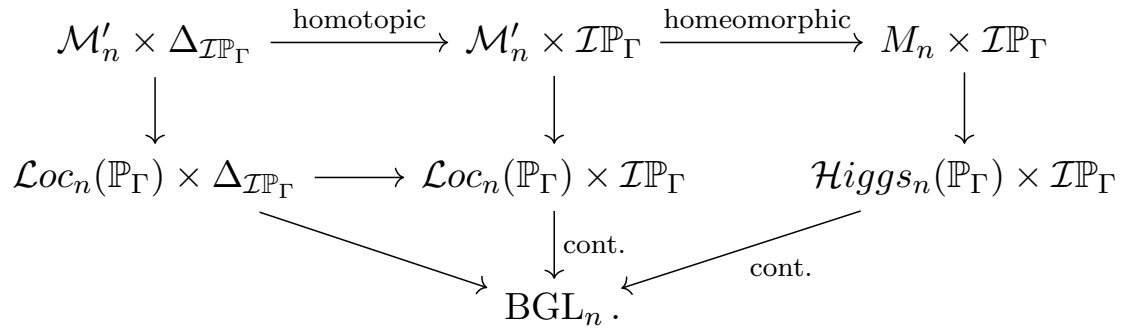

The canonical homeomorphism on the top is induced by the parabolic nonabelian Hodge theorem, and the two continuous maps are induced by the universal families. It follows immediately from the construction that $\mathcal{E}_{n}^{\prime}$ (respectively, $\mathcal{E}_{n}$ ) is the pullback of $\mathrm{EGL}_{n}$ from $\mathrm{BGL}_{n}$ to $\mathcal{M}_{n}^{\prime} \times \mathcal{I P}_{\Gamma}$ (respectively, $M_{n} \times \mathcal{I} \mathbb{P}_{\Gamma}$ ). Therefore, the tautological classes are identified via the canonical homeomorphism $\mathcal{M}_{n}^{\prime} \cong M_{n}$. In particular, statement (i) follows directly from the identification $H^{*}\left(\mathcal{M}_{n}^{\prime}, \mathbb{Q}\right)=H^{*}\left(M_{n}, \mathbb{Q}\right)$ and Theorem 1.3(i).

Now we prove part (ii). The argument in [She17] proves that the morphism $\mathcal{L o c}_{n}\left(\mathbb{P}_{\Gamma}\right) \times \Delta_{\mathcal{I} \mathbb{P}_{\Gamma}} \rightarrow$ $\mathrm{BGL}_{n}$ is algebraic. Note that all the top vertical arrows in the diagrams are forgetful maps, which are also algebraic. Hence we obtain the algebraicity of the morphism

$$
\mathrm{ev}: \mathcal{M}_{n}^{\prime} \times \Delta_{\mathcal{I P}_{\Gamma}} \rightarrow \mathrm{BGL}_{n} \text {. }
$$

Since simplicial schemes carry mixed Hodge structures which are functorial with respect to algebraic morphisms [Del74, Definition 8.3.4, Proposition 8.3.9], we get the following morphism of mixed Hodge structures from (4.18):

$$
H^{*}\left(\mathrm{BGL}_{n}, \mathbb{Q}\right) \rightarrow H^{*}\left(\mathcal{M}_{n}^{\prime}, \mathbb{Q}\right) \otimes H^{*}\left(\Delta_{\mathcal{I P}_{\Gamma}}, \mathbb{Q}\right) .
$$

The cohomology of $\mathrm{BGL}_{n}$ was calculated in [Del74, Theorem 9.1.1],

$$
H^{*}\left(\mathrm{BGL}_{n}, \mathbb{Q}\right)=\bigoplus^{k} \operatorname{Hdg}^{2 k}\left(\mathrm{BGL}_{n}\right),
$$

with ring generators given by the tautological classes $\mathrm{c}_{k}\left(\mathrm{EGL}_{n}\right) \in{ }^{k} \operatorname{Hdg}^{2 k}\left(\mathrm{BGL}_{n}\right)$. Since the group $H^{*}\left(\Delta_{\mathcal{I P}_{\Gamma}}, \mathbb{Q}\right)$ is isomorphic to $H_{\text {orb }}^{*}\left(\mathbb{P}_{\Gamma}, \mathbb{Q}\right)$ as a $\mathbb{Q}$-vector space and carries the Hodge structure in which all classes have weight 0 , we have

$$
\int_{\alpha} \mathrm{c}_{k}\left(\mathcal{E}_{n}^{\prime}\right)=\int_{\alpha} \operatorname{ev}^{*} \mathrm{c}_{k}\left(\mathrm{EGL}_{n}\right) \in{ }^{k} \operatorname{Hdg}^{*}\left(\mathcal{M}_{n}^{\prime}, \mathbb{Q}\right)
$$

for any $\alpha \in H_{\text {orb }}^{*}\left(\mathbb{P}_{\Gamma}, \mathbb{Q}\right)$.

As a corollary, we have the following structural result for the cohomology of $\mathcal{M}_{n}^{\prime}$ which proves (1.9).

Corollary 4.5. The cohomology group of $\mathcal{M}_{n}^{\prime}$ admits a canonical bi-grading decomposition

$$
H^{*}\left(\mathcal{M}_{n}^{\prime}, \mathbb{Q}\right)=\bigoplus_{k, d}{ }^{k} \operatorname{Hdg}^{d}\left(\mathcal{M}_{n}^{\prime}\right) \text {. }
$$




\subsection{Step 5: The $P=W$ conjecture}

Proof of Theorem 1.4. By Theorems 1.3 and 4.4, the tautological classes are the generators of the cohomology ring $H^{*}\left(\mathcal{M}_{n}^{\prime}, \mathbb{Q}\right)=H^{*}\left(M_{n}, \mathbb{Q}\right)$, and they lie in the same piece of the decompositions (1.6) and (1.9). Hence Lemma 4.2 yields the canonical isomorphism of the decompositions

$$
\bigoplus_{k, d} G_{k} H^{d}\left(M_{n}, \mathbb{Q}\right) \stackrel{P=W}{=} \bigoplus_{k, d}^{k} \operatorname{Hdg}^{d}\left(\mathcal{M}_{n}^{\prime}\right)
$$

via the nonabelian Hodge theorem.

\subsection{Applications: Mixed Hodge numbers of character varieties}

For any complex algebraic variety $X$, we define the mixed Hodge polynomial of $X$ as

$$
P(X ; x, y, t)=\sum_{p, q, d} \operatorname{dim}\left(\operatorname{Gr}_{p+q}^{W} H^{d}(X, \mathbb{C})\right)^{p, q} x^{p} y^{q} t^{d} .
$$

The following result follows immediately from Theorem 1.4 and [Zha17, Theorem 5.11], which concerns the generating series of $P\left(\mathcal{M}_{n}^{\prime} ; x, y, t\right)$.

Theorem 4.6. Let $q=x y$. In the $\tilde{A}_{0}$ case, we have

$$
\sum_{n=0}^{\infty} s^{n} P\left(\mathcal{M}_{n}^{\prime} ; x, y, t\right)=\prod_{m=1}^{\infty} \frac{\left(1+s^{m} q^{m} t^{2 m-1}\right)^{2}}{\left(1-s^{m} q^{m-1} t^{2 m-2}\right)\left(1-s^{m} q^{m+1} t^{2 m}\right)} .
$$

In the $\tilde{D}_{4}, \tilde{E}_{6}, \tilde{E}_{7}$, or $\tilde{E}_{8}$ cases, we have

$$
\sum_{n=0}^{\infty} s^{n} P\left(\mathcal{M}_{n}^{\prime} ; x, y, t\right)=\prod_{m=1}^{\infty} \frac{1}{\left(1-s^{m} q^{m-1} t^{2 m-2}\right)\left(1-s^{m} q^{m} t^{2 m}\right)^{K}\left(1-s^{m} q^{m+1} t^{2 m}\right)},
$$

where $K$ is defined in (4.2).

In general, mixed Hodge numbers for character varieties are difficult to compute since the description of a character variety as an affine GIT quotient is not adapted to the computation of mixed Hodge structures. As a corollary of the $P=W$ identity, we are able to obtain closed formulas for the mixed Hodge numbers in the five families of parabolic character varieties using the corresponding perverse filtrations. Our result matches the conjecture proposed by Hausel, Letellier, and Rodriguez-Villegas [HLR11, Conjecture 1.2.1] for the first few values of $n$. (This has been verified numerically by a computer program. However there are still combinatorial difficulties to prove that our formulas coincide with the conjecture in [HLR11] as closed formulas; see [Zha17, Remark 5.13].)

\section{ACKNOWLEDGEMENTS}

We are grateful to Tim-Henrik Bülles, Mark de Cataldo, Davesh Maulik, Rahul Pandharipande, and Qizheng Yin for discussions about Hilbert schemes, perverse filtrations, and Higgs bundles, and to Olivier Biquard for kindly pointing out references. We also thank the anonymous referees for helpful suggestions.

\section{REFERENCES}

BBD82 A. A. Bẹlinson, J. Bernstein, and P. Deligne, Faisceaux pervers, Analysis and Topology on Singular Spaces, I (Luminy, 1981), Astérisque, vol. 100 (Soc. Math. France, Paris, 1982), 5-171. 


\section{J. SHen AND Z. ZHANG}

BGM20 O. Biquard, O. García-Prada, and I. Mundet i Riera, Parabolic Higgs bundles and representations of the fundamental group of a punctured surface into a real group, Adv. Math. 372 (2020), 107305; doi:10.1016/j.aim.2020.107305.

Biq97 O. Biquard, Fibrés de Higgs et connexions intégrables: le cas logarithmique (diviseur lisse), Ann. Sci. École Norm. Sup. (4) 30 (1997), no. 1, 41-96; doi:10.1016/S0012-9593(97)899156.

Che98 J. Cheah, Cellular decompositions for nested Hilbert schemes of points, Pacific J. Math. 183 (1998), no. 1, 39-90; doi:10.2140/pjm.1998.183.39.

deCHM12 M. A. A. de Cataldo, T. Hausel, and L. Migliorini, Topology of Hitchin systems and Hodge theory of character varieties: the case $A_{1}$, Ann. of Math. 175 (2012), no. 3, 1329-1407; doi: 10.4007/annals.2012.175.3.7.

deCHM13_ Exchange between perverse and weight filtration for the Hilbert schemes of points of two surfaces, J. Singul. 7 (2013), 23-38; doi:10.5427/jsing.2013.7c.

deCM02 M. A. A. de Cataldo and L. Migliorini, The Chow groups and the motive of the Hilbert scheme of points on a surface, J. Algebra 251 (2002), no. 2, 824-848; doi:10.1006/jabr.2001.9105.

deCM04 , The Chow motive of semismall resolutions, Math. Res. Lett. 11 (2004), no. 2-3, 151170; doi:10.4310/MRL.2004.v11.n2.a2.

deCM05_, The Hodge theory of algebraic maps, Ann. Sci. École Norm. Sup. (4) 38 (2005), no. 5, 693-750; doi:10.1016/j.ansens.2005.07.001.

deCM09_. The decomposition theorem, perverse sheaves and the topology of algebraic maps, Bull. Amer. Math. Soc. (N.S.) 46 (2009), no. 4, 535-633; doi:10.1090/S0273-0979-09-01260-9.

deCMS19 M.A.A. de Cataldo, D. Maulik, and J. Shen, Hitchin fibrations, abelian surfaces, and the $P=W$ conjecture, 2019, arXiv:1909.11885.

Del74 P. Deligne, Théorie de Hodge. III, Publ. Math. Inst. Hautes Études Sci. (1974), no. 44, 5-77; doi:10.1007/BF02685881.

GNR01 A. Gorsky, N. Nekrasov, and V. Rubtsov, Hilbert schemes, separated variables, and D-branes, Comm. Math. Phys. 222 (2001), no. 2, 299-318; doi:10.1007/s002200100503.

Göt90 L. Göttsche, The Betti numbers of the Hilbert scheme of points on a smooth projective surface, Math. Ann. 286 (1990), no. 1-3, 193-207; doi:10.1007/BF01453572.

Gro14 M. Groechenig, Hilbert schemes as moduli of Higgs bundles and local systems, Int. Math. Res. Not. 2014 (2014), no. 23, 6523-6575; doi:10.1093/imrn/rnt167.

GS93 L. Göttsche and W. Soergel, Perverse sheaves and the cohomology of Hilbert schemes of smooth algebraic surfaces, Math. Ann. 296 (1993), no. 2, 235-245; doi:10.1007/BF01445104.

HLR11 T. Hausel, E. Letellier, and F. Rodriguez-Villegas, Arithmetic harmonic analysis on character and quiver varieties, Duke Math. J. 160 (2011), no. 2, 323-400; doi:10.1215/001270941444258.

HR08 T. Hausel and F. Rodriguez-Villegas, Mixed Hodge polynomials of character varieties (with an appendix by Nicholas M. Katz), Invent. Math. 174 (2008), no. 3, 555-624; doi:10.1007/ s00222-008-0142-x.

HT03 T. Hausel and M. Thaddeus, Relations in the cohomology ring of the moduli space of rank 2 Higgs bundles, J. Amer. Math. Soc. 16 (2003), no. 2, 303-329; doi:10.1090/S0894-0347-0200417-4.

HT04_ Generators for the cohomology ring of the moduli space of rank 2 Higgs bundles, Proc. London Math. Soc. 88 (2004), no. 3, 632-658; doi:10.1112/S0024611503014618.

Leh99 M. Lehn, Chern classes of tautological sheaves on Hilbert schemes of points on surfaces, Invent. Math. 136 (1999), no. 1, 157-207; doi:10.1007/s002220050307.

LQW02 W.-P. Li, Z. Qin, and W. Wang, Vertex algebras and the cohomology ring structure of Hilbert schemes of points on surfaces, Math. Ann. 324 (2002), no. 1, 105-133; doi:10.1007/ s002080200330. 


\section{Perverse filtrations, Hilbert schemes, and $P=W$}

LS03 M. Lehn and C. Sorger, The cup product of Hilbert schemes for K3 surfaces, Invent. Math. 152 (2003), no. 2, 305-329; doi:10.1007/s00222-002-0270-7.

Mar02 E. Markman, Generators of the cohomology ring of moduli spaces of sheaves on symplectic surfaces, J. reine angew. Math. 544 (2002), 61-82; doi:10.1515/crll.2002.028.

Nak97 H. Nakajima, Heisenberg algebra and Hilbert schemes of points on projective surfaces, Ann. of Math. 145 (1997), no. 2, 379-388; doi:10.2307/2951818.

Ols16 M. Olsson, Algebraic spaces and stacks, Amer. Math. Soc. Colloq. Publ., vol. 62 (Amer. Math. Soc., Providence, RI, 2016); doi:10.1090/coll/062.

She17 V. Shende, The weights of the tautological classes of character varieties, Int. Math. Res. Not. 2017 (2017), no. 22, 6832-6840; doi:10.1093/imrn/rnv363.

Sim90 C. T. Simpson, Harmonic bundles on noncompact curves, J. Amer. Math. Soc. 3 (1990), no. 3, 713-770; doi:10.2307/1990935.

Sim92 Higgs bundles and local systems, Publ. Math. Inst. Hautes Études Sci. (1992), no. 75, 5-95; doi:10.1007/BF02699491.

Zha17 Z. Zhang, Multiplicativity of perverse filtration for Hilbert schemes of fibered surfaces, Adv. Math. 312 (2017), 636-679; doi:10.1016/j.aim.2017.03.028.

Junliang Shen jlshen@mit.edu

Department of Mathematics, MIT, Room 2-232C, 77 Massachusetts Avenue, Cambridge, MA 02139, USA

Zili Zhang ziliz@umich.edu

Department of Mathematics, University of Michigan, Room 1821, 530 Church Street, Ann Arbor, MI 48109, USA 